\title{
Instanton effects in ABJM theory from Fermi gas approach
}

\author{
Yasuyuki Hatsuda, ${ }^{a, b}$ Sanefumi Moriyama ${ }^{c}$ and Kazumi Okuyama ${ }^{d}$ \\ ${ }^{a}$ DESY Theory Group, DESY Hamburg, \\ Notkestrasse 85, D-22603 Hamburg, Germany \\ ${ }^{b}$ Department of Physics, Tokyo Institute of Technology, \\ Tokyo 152-8551, Japan \\ ${ }^{c}$ Kobayashi Maskawa Institute $\&$ Graduate School of Mathematics, Nagoya University, \\ Nagoya 464-8602, Japan \\ ${ }^{d}$ Department of Physics, Shinshu University, \\ Matsumoto 390-8621, Japan \\ E-mail: yasuyuki.hatsuda@desy.de, moriyama@math.nagoya-u.ac.jp, \\ kazumi@azusa.shinshu-u.ac.jp
}

ABstract: We study the instanton effects of the ABJM partition function using the Fermi gas formalism. We compute the exact values of the partition function at the Chern-Simons levels $k=1,2,3,4,6$ up to $N=44,20,18,16,14$ respectively, and extract non-perturbative corrections from these exact results. Fitting the resulting non-perturbative corrections by their expected forms from the Fermi gas, we determine unknown parameters in them. After separating the oscillating behavior of the grand potential, which originates in the periodicity of the grand partition function, and the worldsheet instanton contribution, which is computed from the topological string theory, we succeed in proposing an analytical expression for the leading D2-instanton correction. Just as the perturbative result, the instanton corrections to the partition function are expressed in terms of the Airy function.

KeYwords: Matrix Models, Nonperturbative Effects, Chern-Simons Theories, M-Theory

ARXIV EPRINT: 1211.1251 


\section{Contents}

1 Introduction $\quad 1$

2 Exact results of partition function 5

3 Numerical study $\quad 8$

$\begin{array}{ll}3.1 \text { Grand potential } & 8\end{array}$

$\begin{array}{lr}3.2 \text { Oscillations in } J_{k}(\mu) & 10\end{array}$

$\begin{array}{lll}3.3 & \text { Partition function } & 13\end{array}$

4 Worldsheet instantons $\quad 16$

$\begin{array}{ll}4.1 \text { Topological string on local } \mathbb{F}_{0} & 16\end{array}$

$\begin{array}{ll}\text { 4.2 Worldsheet instanton in the 't Hooft limit } & 18\end{array}$

5 Conjecture of D2-instanton corrections $\quad 20$

5.1 D2 1-instanton correction 20

5.2 Comment on D2 2-instanton correction 22

$\begin{array}{lll}6 & \text { Discussions } & 23\end{array}$

A Proof of (2.22) for general Hankel matrices $\quad \mathbf{2 4}$

A.1 Proof of the even part 25

$\begin{array}{lll}\text { A.2 Proof of the odd part } & 27\end{array}$

B Exact values of $Z_{k}(N) \quad 28$

$\begin{array}{lll}\text { B.1 Recursive relations at even } k & 28\end{array}$

$\begin{array}{lll}\text { B.2 Recursive relations at odd } k & 31\end{array}$

B.3 A guess 32

\section{Introduction}

Partition functions are the most fundamental quantities in both quantum systems and statistical systems. Although it typically serves only the role of normalization in the physical interpretation, it encodes important thermodynamic information such as the free energy. In field theories, the partition function, in general, contains the information of not only perturbation but also instanton effects as well as some global analytical structures. Therefore it is natural to study it as a first step.

M-theory is supposed to unify all our understandings of non-perturbative effects in the string theory. In the seminal paper [1], the low-energy effective theory on $N$ multiple 
M2-branes on the geometry $\mathbb{C}^{4} / \mathbb{Z}_{k}$ was proposed to be the $\mathcal{N}=6$ supersymmetric ChernSimons theory with gauge group $\mathrm{U}(N)_{k} \times \mathrm{U}(N)_{-k}$ and bifundamental matters $A_{1,2}, B_{1,2}$ forming the superpotential $W=\frac{2 \pi}{k}\left(A_{1} B_{1} A_{2} B_{2}-A_{1} B_{2} A_{2} B_{1}\right)$.

It was found in [2-4] that the infinite-dimensional path integral of the partition function on $S^{3}$ and the expectation values of BPS Wilson loops are reduced to a finite dimensional matrix model by the localization techniques [5].

There is much progress in this matrix model including the derivation of the degree of freedom $N^{3 / 2}[6]$ expected from the AdS/CFT correspondence [7] and its perturbative completion by the Airy function [8]. Recently, the ABJM partition function was rewritten as that of a Fermi gas system [9-11], where the standard method in statistical mechanics is applicable and the above properties are reproduced easily.

The Airy function and the Fermi gas formalism imply a deep structure of M-theory. The Airy function has the integral representation of the exponentiated cubic term, which looks similar to the partition function of the Chern-Simons theory, and appears also in other contexts of M-theory [12]. Therefore we would like to study the partition function further and to extract some physical information out of it.

In our previous work [13], we have proposed a method to compute the exact partition function $Z_{k}(N)$ at $k=1$ and indeed computed it up to $N=9$. Here with a generalization of our method and a technique from [14], we perform the computation of the exact partition function at $k=1,2,3,4,6$ up to $N=44,20,18,16,14$ respectively.

After the computation of the exact values, we proceed to study the non-perturbative effects. We can extract the non-perturbative corrections from the exact values of the partition function by subtracting the perturbative result. The main motivation of this paper is to explore an analytic form of the non-perturbative correction. It is known that there are two kinds of instantons that induce the non-perturbative corrections to the ABJM partition function. One is called the worldsheet instanton, and the other the D2-instanton (membrane instanton). From the viewpoint of the gravity dual, the Type IIA string theory on $\mathrm{AdS}_{4} \times \mathbb{C P}^{3}$, the worldsheet instanton comes from the fundamental string wrapping the holomorphic cycle $\mathbb{C P}^{1} \subset \mathbb{C P}^{3}[6,15]$, while the D2-instanton comes from the D2-brane wrapping the Lagrangian submanifold $\mathbb{R P}^{3} \subset \mathbb{C P}^{3}[16]$. The worldsheet instanton behaves as $e^{-2 \pi \sqrt{2 N / k}}$ while the D2-instanton as $e^{-\pi \sqrt{2 k N}}$ in the large $N$ limit.

Let us summarize our results here. Using the Fermi gas formalism (and some results of the topological string), we can know the expected forms of the instanton corrections to the partition function. In the Fermi gas formalism, it is useful to consider the grand partition function. The grand potential (the logarithm of the grand partition function), in general, receives the non-perturbative corrections as

$$
J_{k}^{(\mathrm{np})}(\mu) \equiv J_{k}(\mu)-J_{k}^{(\mathrm{pert})}(\mu)=J_{k}^{\mathrm{D} 2}(\mu)+J_{k}^{\mathrm{WS}}(\mu)+\cdots,
$$

where $\mu$ is the chemical potential, and $J_{k}^{(\text {pert })}(\mu), J_{k}^{\mathrm{D} 2}(\mu)$ and $J_{k}^{\mathrm{WS}}(\mu)$ are the perturbative, D2-instanton and worldsheet instanton contributions, respectively. We note that the dots in (1.1) represent an additional oscillatory contribution and, if any, bound states of the worldsheet instanton and D2-instanton. The oscillatory behavior will be studied in subsec- 
tion 3.2 in detail, which we ignore here. The D2-instanton and the worldsheet instanton corrections are expected to take the following forms:

$$
\begin{aligned}
& J_{k}^{\mathrm{D} 2}(\mu)=\sum_{m=1}^{\infty} J_{k}^{\mathrm{D} 2(m)}(\mu)=\sum_{m=1}^{\infty}\left[a_{k}^{(m)} \mu^{2}+b_{k}^{(m)} \mu+c_{k}^{(m)}\right] e^{-2 m \mu}, \\
& J_{k}^{\mathrm{WS}}(\mu)=\sum_{n=1}^{\infty} J_{k}^{\mathrm{WS}(n)}(\mu)=\sum_{n=1}^{\infty} d_{k}^{(n)} e^{-\frac{4 n \mu}{k}} .
\end{aligned}
$$

We would like to determine the prefactors of those instanton corrections. The prefactors of the worldsheet instanton corrections are determined by the results of the topological string on local $\mathbb{F}_{0}$, where the first few prefactors are

$$
\begin{aligned}
& d_{k}^{(1)}=\frac{1}{\sin ^{2}\left(\frac{2 \pi}{k}\right)}, \quad d_{k}^{(2)}=-\frac{1}{2 \sin ^{2}\left(\frac{4 \pi}{k}\right)}-\frac{1}{\sin ^{2}\left(\frac{2 \pi}{k}\right)}, \quad d_{k}^{(3)}=\frac{1}{3 \sin ^{2}\left(\frac{6 \pi}{k}\right)}+\frac{3}{\sin ^{2}\left(\frac{2 \pi}{k}\right)}, \\
& d_{k}^{(4)}=9-\frac{12}{\sin ^{2}\left(\frac{2 \pi}{k}\right)}-\frac{1}{2 \sin ^{2}\left(\frac{4 \pi}{k}\right)}-\frac{1}{4 \sin ^{2}\left(\frac{8 \pi}{k}\right)} .
\end{aligned}
$$

On the other hand, there are no systematic ways to determine the prefactors of the D2instanton corrections. ${ }^{1}$ In this paper, we propose the analytic form of the leading D2instanton correction,

$$
\begin{aligned}
& a_{k}^{(1)}=-\frac{4}{\pi^{2} k} \cos \left(\frac{\pi k}{2}\right), \quad b_{k}^{(1)}=\frac{2}{\pi \tan \left(\frac{\pi k}{2}\right)} \cos \left(\frac{\pi k}{2}\right), \\
& c_{k}^{(1)}=\left[-\frac{2}{3 k}+\frac{5 k}{12}+\frac{k}{2 \sin ^{2}\left(\frac{\pi k}{2}\right)}+\frac{1}{\pi \tan \left(\frac{\pi k}{2}\right)}\right] \cos \left(\frac{\pi k}{2}\right) .
\end{aligned}
$$

We arrive at these expressions from many constraints (by matching the exact data, the numerical data from TBA, pole structure, small $k$ behavior etc.) and a few assumptions. We believe that these results are valid for any $k$. We also find the higher instanton corrections at $k=1,2,3,4,6$ from the numerical fitting. Here is the summary of our results:

$$
\begin{aligned}
J_{1}^{(\mathrm{np})}= & {\left[\frac{4 \mu^{2}+\mu+1 / 4}{\pi^{2}}\right] e^{-4 \mu}+\left[-\frac{52 \mu^{2}+\mu / 2+9 / 16}{2 \pi^{2}}+2\right] e^{-8 \mu} } \\
& +\left[\frac{736 \mu^{2}-152 \mu / 3+77 / 18}{3 \pi^{2}}-32\right] e^{-12 \mu} \\
& +\left[-\frac{2701 \mu^{2}-13949 \mu / 48+11291 / 768}{\pi^{2}}+466\right] e^{-16 \mu}+\mathcal{O}\left(e^{-20 \mu}\right), \\
J_{2}^{(\mathrm{np})}= & {\left[\frac{4 \mu^{2}+2 \mu+1}{\pi^{2}}\right] e^{-2 \mu}+\left[-\frac{52 \mu^{2}+\mu+9 / 4}{2 \pi^{2}}+2\right] e^{-4 \mu} } \\
& +\left[\frac{736 \mu^{2}-304 \mu / 3+154 / 9}{3 \pi^{2}}-32\right] e^{-6 \mu} \\
& +\left[-\frac{2701 \mu^{2}-13949 \mu / 24+11291 / 192}{\pi^{2}}+466\right] e^{-8 \mu}+\mathcal{O}\left(e^{-10 \mu}\right),
\end{aligned}
$$

\footnotetext{
${ }^{1}$ One approach is to consider the 'WKB' (small $k$ ) expansions of the prefactors as in [9]. However, it is hard to determine the higher order corrections in this way.
} 


$$
\begin{aligned}
& J_{3}^{(\mathrm{np})}=\frac{4}{3} e^{-\frac{4}{3} \mu}-2 e^{-\frac{8}{3} \mu}+\left[\frac{4 \mu^{2}+\mu+1 / 4}{3 \pi^{2}}+\frac{20}{9}\right] e^{-4 \mu}-\frac{88}{9} e^{-\frac{16}{3} \mu}+\mathcal{O}\left(e^{-\frac{20}{3} \mu}\right), \\
& J_{4}^{(\mathrm{np})}=e^{-\mu}+\left[-\frac{4 \mu^{2}+2 \mu+1}{2 \pi^{2}}\right] e^{-2 \mu}+\frac{16}{3} e^{-3 \mu}+\left[-\frac{52 \mu^{2}+\mu+9 / 4}{4 \pi^{2}}+2\right] e^{-4 \mu}+\mathcal{O}\left(e^{-5 \mu}\right), \\
& J_{6}^{(\mathrm{np})}=\frac{4}{3} e^{-\frac{2}{3} \mu}-2 e^{-\frac{4}{3} \mu}+\left[\frac{4 \mu^{2}+2 \mu+1}{3 \pi^{2}}+\frac{20}{9}\right] e^{-2 \mu}-\frac{88}{9} e^{-\frac{8}{3} \mu}+\mathcal{O}\left(e^{-\frac{10}{3} \mu}\right) .
\end{aligned}
$$

As mentioned above, besides (1.5) there are additional oscillatory terms whose explicit forms can be found in subsection 3.1.

Once the instanton corrections to the grand potential are fixed, we can easily translate them into the instanton corrections to the partition function,

$$
Z_{k}^{(\mathrm{np})}(N) \equiv \frac{Z_{k}^{(\text {exact })}(N)}{Z_{k}^{(\text {pert })}(N)}-1,
$$

where $Z_{k}^{(\text {pert) }}(N)$ is the perturbative result in [8], whose explicit form is given by (3.18). The leading corrections of both instantons are written by the Airy function (and its derivative):

$$
\begin{aligned}
Z_{k}^{\mathrm{D} 2(1)}(N)= & \left(C_{k}^{-1}\left(N+2-B_{k}\right) a_{k}^{(1)}+c_{k}^{(1)}\right) \frac{\mathrm{Ai}\left[C_{k}^{-1 / 3}\left(N+2-B_{k}\right)\right]}{\operatorname{Ai}\left[C_{k}^{-1 / 3}\left(N-B_{k}\right)\right]} \\
& -C_{k}^{-1 / 3} b_{k}^{(1)} \frac{\mathrm{Ai}^{\prime}\left[C_{k}^{-1 / 3}\left(N+2-B_{k}\right)\right]}{\operatorname{Ai}\left[C_{k}^{-1 / 3}\left(N-B_{k}\right)\right]} \\
Z_{k}^{\mathrm{WS}(1)}(N)= & d_{k}^{(1)} \frac{\operatorname{Ai}\left[C_{k}^{-1 / 3}\left(N+\frac{4}{k}-B_{k}\right)\right]}{\operatorname{Ai}\left[C_{k}^{-1 / 3}\left(N-B_{k}\right)\right]}
\end{aligned}
$$

with

$$
B_{k}=\frac{k}{24}+\frac{1}{3 k}, \quad C_{k}=\frac{2}{\pi^{2} k} .
$$

Some remarks on our results are mentioned in order.

Firstly, we emphasize that our results include all the $1 / N$ corrections. One can check that in the large $N$ limit, both instanton corrections show the correct exponentially suppressed behavior as mentioned above.

Secondly, the worldsheet instanton correction (1.8) is divergent at $k=1,2$, and the D2-instanton correction (1.7) is also divergent for even $k .^{2}$ Therefore we cannot apply the above result to these values of $k$. In these cases, the D2-instanton and worldsheet instanton corrections are mixed, and the sum of them totally become finite. At $k=1,2$, for example, such mixed instanton corrections are given by

$$
\begin{aligned}
& Z_{1}^{\mathrm{D} 2+\mathrm{WS}}(N)=\left(2 N+\frac{C_{1}}{8}+\frac{29}{4}\right) \frac{\mathrm{Ai}\left[C_{1}^{-1 / 3}\left(N+\frac{29}{8}\right)\right]}{\operatorname{Ai}\left[C_{1}^{-1 / 3}\left(N-\frac{3}{8}\right)\right]}-\frac{C_{1}^{2 / 3}}{2} \frac{\mathrm{Ai}^{\prime}\left[C_{1}^{-1 / 3}\left(N+\frac{29}{8}\right)\right]}{\operatorname{Ai}\left[C_{1}^{-1 / 3}\left(N-\frac{3}{8}\right)\right]}, \\
& Z_{2}^{\mathrm{D} 2+\mathrm{WS}}(N)=\left(4 N+C_{2}+7\right) \frac{\operatorname{Ai}\left[C_{2}^{-1 / 3}\left(N+\frac{7}{4}\right)\right]}{\operatorname{Ai}\left[C_{2}^{-1 / 3}\left(N-\frac{1}{4}\right)\right]}-2 C_{2}^{2 / 3} \frac{\operatorname{Ai}^{\prime}\left[C_{2}^{-1 / 3}\left(N+\frac{7}{4}\right)\right]}{\operatorname{Ai}\left[C_{2}^{-1 / 3}\left(N-\frac{1}{4}\right)\right]}
\end{aligned}
$$

\footnotetext{
${ }^{2}$ For odd $k$, the D2-instanton correction (1.7) vanishes, and the leading correction starts from $\mathcal{O}\left(e^{-2 \pi \sqrt{2 k N}}\right)$.
} 
One can check that the leading large $N$ behavior of (1.10) precisely reproduces the result in [14]. We note again that our results include all the $1 / N$ corrections.

Finally, for $k>2$, the leading non-perturbative contribution comes from the worldsheet instanton. Since our result is valid for any $N$ and $k$, we can take the 't Hooft limit. Our worldsheet instanton correction (1.8) must give the correct answer in this limit. We check that the genus-zero and genus-one worldsheet instanton corrections to the free energy studied in [6] are indeed reproduced from our result (1.8) after taking the 't Hooft limit. This is a non-trivial check of our result.

The paper is organized as follows. In section 2, we review the method to compute the partition function exactly. We generalize our previous method in [13] to arbitrary level $k$. In section 3, using the exact partition function obtained by this method, we numerically study the behavior of the instanton corrections. We focus on the grand potential and the partition function. We find that the grand potential shows an oscillatory behavior and that it is explained by the periodicity of the grand partition function. By the numerical fitting, we determine the instanton corrections to the grand potential at $k=1,2,3,4,6$. In section 4, we compute the worldsheet instanton corrections by using the results in the topological string on local $\mathbb{F}_{0}$. As a non-trivial test, we take the 't Hooft limit of our results, and confirm that our results correctly reproduce the worldsheet instanton corrections to the genus-zero and genus-one free energies up to 3 -instanton. In section 5 , we propose an analytic form of the D2-instanton correction, and check its validity. Section 6 is devoted to conclusions and discussions. In appendix A, we prove a new relation found in this paper. In appendix B, we summarize the exact values of the partition function at $k=1,2,3,4,6$.

\section{Exact results of partition function}

In [9] the ABJM partition function

$$
Z_{k}(N)=\frac{1}{(N !)^{2}} \int \frac{d^{N} x_{i}}{(2 \pi)^{N}} \frac{d^{N} y_{i}}{(2 \pi)^{N}} \frac{\prod_{i<j}\left[2 \sinh \frac{\mu_{i}-\mu_{j}}{2}\right]^{2}\left[2 \sinh \frac{\nu_{i}-\nu_{j}}{2}\right]^{2}}{\prod_{i, j}\left[2 \cosh \frac{\mu_{i}-\nu_{j}}{2}\right]^{2}} \exp \left[\frac{i k}{4 \pi} \sum_{i}\left(\mu_{i}^{2}-\nu_{i}^{2}\right)\right]
$$

is rewritten in terms of the partition function of a Fermi gas system

$$
Z_{k}(N)=\frac{1}{N !} \sum_{\sigma \in S_{N}}(-1)^{\epsilon(\sigma)} \int \frac{d^{N} q}{(2 \pi)^{N}} \prod_{i} \rho\left(q_{i}, q_{\sigma(i)}\right),
$$

with the density matrix given by

$$
\rho\left(q_{1}, q_{2}\right)=\frac{1}{k} \frac{1}{\sqrt{2 \cosh \frac{q_{1}}{2}}} \frac{1}{2 \cosh \frac{q_{1}-q_{2}}{2 k}} \frac{1}{\sqrt{2 \cosh \frac{q_{2}}{2}}} .
$$

After introducing the chemical potential $\mu$ or the fugacity $z=e^{\mu}$, the grand partition function

$$
\Xi_{k}(\mu)=1+\sum_{N=1}^{\infty} Z_{k}(N) e^{\mu N}
$$


is given by

$$
\Xi_{k}(\mu)=\operatorname{det}(1+z \rho)=\exp \left[-\sum_{n=1}^{\infty} \frac{(-z)^{n}}{n} \operatorname{Tr} \rho^{n}\right] .
$$

This means that if we want to know the partition functions for various $N$, all we have to do is to compute $\operatorname{Tr} \rho^{n}$ for $n=1, \cdots, N$.

As explained previously in [13], it is difficult to compute $\operatorname{Tr} \rho^{n}$ because of the complexity in convoluting the density matrix. This difficulty was, however, overcome by a lemma from [17] stating that when a kernel $K\left(q_{1}, q_{2}\right)$ takes the form

$$
K\left(q_{1}, q_{2}\right)=\frac{E\left(q_{1}\right) E\left(q_{2}\right)}{M\left(q_{1}\right)+M\left(q_{2}\right)},
$$

we can compute its power $K^{n}\left(q_{1}, q_{2}\right)$ by

$$
K^{n}\left(q_{1}, q_{2}\right)=\frac{E\left(q_{1}\right) E\left(q_{2}\right)}{M\left(q_{1}\right)+(-1)^{n-1} M\left(q_{2}\right)} \sum_{\ell=0}^{n-1}(-1)^{\ell} \phi^{\ell}\left(q_{1}\right) \phi^{n-1-\ell}\left(q_{2}\right),
$$

where $\phi^{\ell}(q)$ is defined by

$$
\phi^{\ell}(q)=\frac{1}{E(q)} \int \frac{d q^{\prime}}{2 \pi} K^{\ell}\left(q, q^{\prime}\right) E\left(q^{\prime}\right) .
$$

We can compute $\phi^{\ell}(q)$ recursively

$$
\phi^{\ell}(q)=\frac{1}{E(q)} \int \frac{d q^{\prime}}{2 \pi} K\left(q, q^{\prime}\right) E\left(q^{\prime}\right) \phi^{\ell-1}\left(q^{\prime}\right),
$$

with the initial condition $\phi^{0}(q)=1$. Though we can apply this method to the density matrix $\rho$ directly, there is a more convenient way to compute the grand partition function as explained in [13].

Let us briefly review the method found in [13]. As our density matrix is invariant under the parity

$$
\rho\left(-q_{1},-q_{2}\right)=\rho\left(q_{1}, q_{2}\right)
$$

we can decompose it into two parts:

$$
\rho\left(q_{1}, q_{2}\right)=\rho_{+}\left(q_{1}, q_{2}\right)+\rho_{-}\left(q_{1}, q_{2}\right), \quad \rho_{ \pm}\left(q_{1}, q_{2}\right) \equiv \frac{\rho\left(q_{1}, q_{2}\right) \pm \rho\left(q_{1},-q_{2}\right)}{2} .
$$

Since $\rho_{+}$(and also $\rho_{-}$) is written as the form (2.6),

$$
\rho_{+}\left(q_{1}, q_{2}\right)=\frac{E\left(q_{1}\right) E\left(q_{2}\right)}{\cosh \left(\frac{q_{1}}{k}\right)+\cosh \left(\frac{q_{2}}{k}\right)},
$$

with

$$
E(q)=\frac{\cosh \left(\frac{q}{2 k}\right)}{\sqrt{2 k \cosh \left(\frac{q}{2}\right)}}
$$


we can compute $\rho_{+}^{n}$ from a series of functions $\phi^{\ell}(q)$ as

$$
\rho_{+}^{n}\left(q_{1}, q_{2}\right)=\frac{E\left(q_{1}\right) E\left(q_{2}\right)}{\cosh \left(\frac{q_{1}}{k}\right)+(-1)^{n-1} \cosh \left(\frac{q_{2}}{k}\right)} \sum_{\ell=0}^{n-1}(-1)^{\ell} \phi^{\ell}\left(q_{1}\right) \phi^{n-1-\ell}\left(q_{2}\right) .
$$

The important fact is that using (2.9) the functions $\phi^{\ell}(q)$ are determined by the following recursive relation:

$$
\phi^{\ell}(q)=\frac{1}{2 k \cosh \left(\frac{q}{2 k}\right)} \int_{-\infty}^{\infty} \frac{d q^{\prime}}{2 \pi} \frac{1}{2 \cosh \left(\frac{q-q^{\prime}}{2 k}\right)} \frac{\cosh \left(\frac{q^{\prime}}{2 k}\right)}{\cosh \left(\frac{q^{\prime}}{2}\right)} \phi^{\ell-1}\left(q^{\prime}\right) .
$$

One can show that the grand partition function is factorized as

$$
\Xi(\mu)=\operatorname{det}\left(1+z \rho_{+}\right) \operatorname{det}\left(1+z \rho_{-}\right) .
$$

In [13], we found that the density matrices enjoy an interesting property for $k=1$,

$$
\frac{\operatorname{det}\left(1+z \rho_{-}\right)}{\operatorname{det}\left(1-z \rho_{+}\right)}=\sum_{\ell=0}^{\infty} \phi^{\ell}(0) z^{\ell}
$$

Thus the grand partition function is rewritten as

$$
\Xi(\mu)=\operatorname{det}\left(1-z^{2} \rho_{+}^{2}\right) \cdot \sum_{\ell=0}^{\infty} \phi^{\ell}(0) z^{\ell},
$$

and determined only by the even parity functions $\phi^{\ell}(q)$ in this case.

In [13], we further found that the eigenvalue problem of the density matrix $\rho$ is mapped to that of an infinite-dimensional parity-preserving Hankel matrix, whose form is given by

$$
H=\left(\begin{array}{ccccccc}
h_{0} & 0 & h_{1} & 0 & h_{2} & 0 & \\
0 & h_{1} & 0 & h_{2} & 0 & h_{3} & \\
h_{1} & 0 & h_{2} & 0 & h_{3} & 0 & \ldots \\
0 & h_{2} & 0 & h_{3} & 0 & h_{4} & \\
h_{2} & 0 & h_{3} & 0 & h_{4} & 0 & \\
0 & h_{3} & 0 & h_{4} & 0 & h_{5} & \\
& \vdots & & & & \ddots
\end{array}\right)
$$

Thus the grand partition function is also expressed as

$$
\Xi(\mu)=\operatorname{det}(1+z \rho)=\operatorname{det}(1+z H) .
$$

In this paper, we find a formula for arbitrary parity-preserving Hankel matrices, which reduces to the same expression (2.17) for a general value of $k$. Let $H_{ \pm}$be the even/odd parity block of the original Hankel matrix,

$$
H_{+}=\left(\begin{array}{cccc}
h_{0} & h_{1} & h_{2} & \\
h_{1} & h_{2} & h_{3} & \cdots \\
h_{2} & h_{3} & h_{4} & \\
\vdots & & \ddots
\end{array}\right), \quad H_{-}=\left(\begin{array}{cccc}
h_{1} & h_{2} & h_{3} & \\
h_{2} & h_{3} & h_{4} & \cdots \\
h_{3} & h_{4} & h_{5} & \\
\vdots & & \ddots
\end{array}\right)
$$


Then it satisfies the following relation

$$
\frac{\operatorname{det}\left(1+z H_{-}\right)}{\operatorname{det}\left(1-z H_{+}\right)}=\left\langle e_{0}\left|\frac{1}{1-z H_{+}}\right| v\right\rangle,
$$

with

$$
\left|e_{0}\right\rangle=(1,0,0, \cdots)^{\mathrm{T}}, \quad|v\rangle=(1,1,1, \cdots)^{\mathrm{T}} .
$$

In appendix A, we shall give a proof of (2.22) for a certain class of Hankel matrices.

Using these methods we computed the partition functions at $k=1,2,3,4,6$. The exact values are given in appendix B. As can be imagined from [18], the partition functions have close relations with $\tan \left(\frac{\pi}{k}\right)$. Therefore it is especially clean for these values of $k$.

\section{Numerical study}

As summarized in appendix $\mathrm{B}$, we obtain the exact values of the partition functions at $k=$ $1,2,3,4,6$ up to $N_{\max }=44,20,18,16,14$, respectively, following the procedure in section 2 . After computing the exact partition function, let us extract important information from it. Here we numerically study the non-perturbative corrections to the partition function. We shall plot various physical quantities computed by our exact values and fit them by the theoretically expected forms $[6,9,16]$.

\subsection{Grand potential}

First, we start with the grand potential $J_{k}(\mu)$ defined by

$$
J_{k}(\mu)=\log \Xi_{k}(\mu) .
$$

From the theoretical expectation the prefactor of the instanton corrections are at most quadratic functions of $\mu$ (see (1.2)). In fact, we find numerically that the grand potential $J_{k}(\mu)(k=1,2,3,4,6)$ generally can be expanded $\operatorname{as}^{3}$

$$
J_{k}(\mu)=J_{k}^{(\text {pert })}(\mu)+\sum_{n=1}^{\infty} J_{k}^{(n)} e^{-\frac{4 n \mu}{k}}, \quad J_{k}^{(n)}=\alpha_{k}^{(n)} \mu^{2}+\beta_{k}^{(n)} \mu+\gamma_{k}^{(n)} .
$$

In the following we shall plot the grand potential and their instanton corrections, fit them by the quadratic functions and read off the coefficients numerically.

However, not all of the coefficients are read off from this analysis of $J_{k}(\mu)$. As we shall explain below we will encounter some oscillations in $\mu$ and it is difficult to study the exact values of the coefficients due to large errors caused by the oscillations. Some of the values are actually first read off from the analysis of the partition function $Z_{k}(N)$ as in subsection 3.3 which does not have the oscillating behavior, while some of them are found after we understand the oscillating behavior well as in subsection 3.2 and subtract its contribution. It is simpler, however, to present our result as if we read all of the exact values from the grand potential and explain various behaviors later. Hence, we shall take this path in presenting our results.

\footnotetext{
${ }^{3}$ We expect that this property holds for $k \in \mathbb{Z}$. However, we cannot establish it at present.
} 


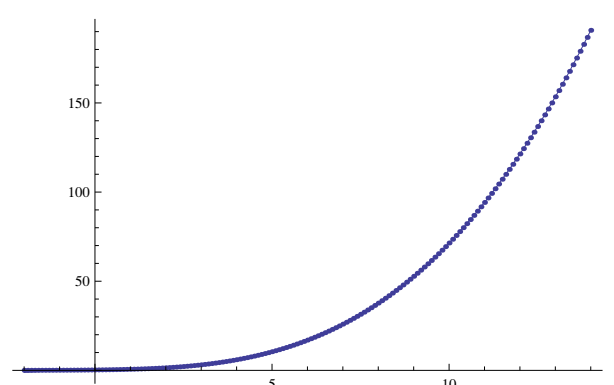

(a)

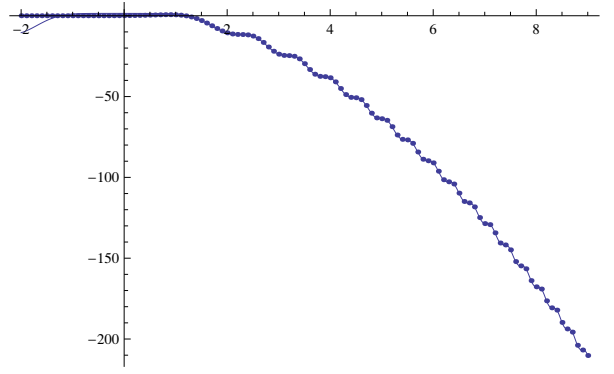

(c)

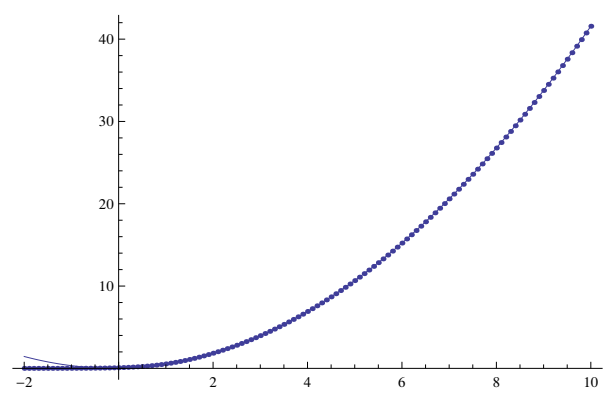

(b)

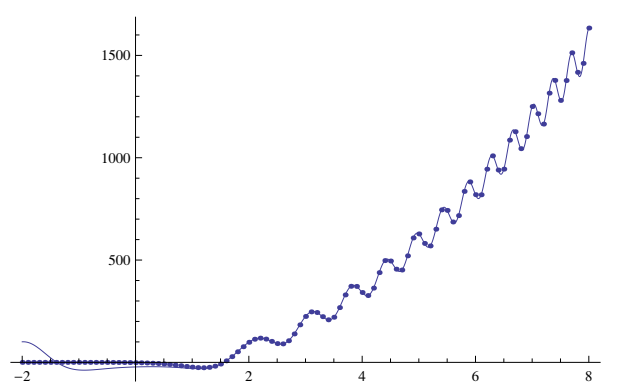

(d)

Figure 1. (a) The perturbative, (b) 1-instanton, (c) 2-instanton and (d) 3-instanton corrections of the grand potential $J_{k}(\mu)$ at $k=1$. The dots represent the grand potential obtained by the exact partition function while the solid lines represent the fitted functions.

Let us start to study the grand potential at $k=1$. In figure 1(a), we have plotted the grand potential $J_{1}(\mu)$ (for selected values of $\mu$ ) in dots together with its perturbative expression $J_{1}^{(\text {pert })}(\mu)$ in a solid line. The grand potential $J_{k}(\mu)(3.1)$ is given by the grand partition function $\Xi_{k}(\mu)(2.4)$ with the summation of $N$ truncated at our maximal values $N_{\max }$, while the perturbative expression is given by

$$
J_{k}^{(\text {pert })}(\mu)=\frac{C_{k}}{3} \mu^{3}+B_{k} \mu+A_{k} .
$$

Here the constants $B_{k}$ and $C_{k}$ are given by (1.9), and $A_{k}$ was conjectured in $[9,19]$ as

$$
A_{k}=-\frac{\zeta(3)}{8 \pi^{2}} k^{2}+\frac{1}{6} \log \frac{4 \pi}{k}+2 \zeta^{\prime}(-1)-\frac{1}{3} \int_{0}^{\infty} d x \frac{1}{e^{k x}-1}\left(\frac{3}{x^{3}}-\frac{1}{x}-\frac{3}{x \sinh ^{2} x}\right) .
$$

We find a perfect match, especially for large $\mu$. Therefore, we can plot $J_{1}^{(1)}(\mu)=\left(J_{1}(\mu)-\right.$ $\left.J_{1}^{(\text {pert })}(\mu)\right) / e^{-4 \mu}$ next in figure 1 (b) and fit the result by a quadratic function. We find the behavior matches well with

$$
J_{1}^{(1)}(\mu)=\frac{4 \mu^{2}+\mu+1 / 4}{\pi^{2}} .
$$

We continue this process further and plot $J_{1}^{(2)}(\mu)=\left(J(\mu)-J_{1}^{(\text {pert })}(\mu)-e^{-4 \mu} J_{1}^{(1)}(\mu)\right) / e^{-8 \mu}$ in figure 1(c). Now the plot starts to oscillate. We can fit the result by the sum of a 
quadratic function with the oscillations $\cos \left(\frac{4 \mu^{2}}{\pi}\right)$ and $\sin \left(\frac{4 \mu^{2}}{\pi}\right)$,

$$
\begin{aligned}
J_{1}^{(2)}(\mu) & =-\frac{52 \mu^{2}+\mu / 2+9 / 16}{2 \pi^{2}}+2+J_{1}^{(2) \mathrm{osc}}(\mu), \\
J_{1}^{(2) \mathrm{osc}}(\mu) & =2 \cos \left(\frac{4 \mu^{2}}{\pi}-\frac{7 \pi}{12}\right) .
\end{aligned}
$$

Similarly, the 3-instanton $J_{1}^{(3)}(\mu)=\left(J_{1}(\mu)-J_{1}^{(\text {pert })}(\mu)-e^{-4 \mu} J_{1}^{(1)}(\mu)-e^{-8 \mu} J_{1}^{(2)}(\mu)\right) / e^{-12 \mu}$ is plotted in figure $1(\mathrm{~d})$, and the fitted function results in

$$
\begin{aligned}
J_{1}^{(3)}(\mu) & =\frac{736 \mu^{2}-152 \mu / 3+77 / 18}{3 \pi^{2}}-32+J_{1}^{(3) \text { osc }}(\mu), \\
J_{1}^{(3) \text { osc }}(\mu) & =-\frac{4}{\pi}(8 \mu+1) \sin \left(\frac{4 \mu^{2}}{\pi}-\frac{7 \pi}{12}\right)-32 \cos \left(\frac{4 \mu^{2}}{\pi}-\frac{7 \pi}{12}\right) .
\end{aligned}
$$

Note that our plot range of $\mu$ changes slightly. This is because in going to higher and higher instanton effects, we have more and more numerical errors and it is difficult to find a sensible plot for large $\mu$.

We can repeat the same analysis at $k=2,3,4,6$ since we have exact values. The results computed from the exact values we find are plotted in figures $2,3,4,5$, respectively together with the fitted functions. The results are summarized in (1.5) (we have suppressed the oscillatory parts in (1.5)). Using the results in (1.5), we can study various instanton effects. This will be done in the subsequent sections. Before proceeding to it, let us give a physical interpretation for the oscillating behaviors and present the numerical study of $Z(N)$ without the oscillations.

\subsection{Oscillations in $J_{k}(\mu)$}

In this subsection, we would like to explain the oscillation in $J_{k}(\mu)$ from the periodicity of the grand partition function. Since the grand partition function $\Xi_{k}(\mu)=e^{J_{k}(\mu)}$ is a sum of $e^{N \mu}$ defined by (2.4), it must be a periodic function of $\mu$ with the periodicity $2 \pi i$. However, the naive expression of $\Xi_{k}(\mu)$ derived from the method of statistical mechanics in small $k$ perturbation is not invariant under the shift $\mu \rightarrow \mu+2 \pi i$.

To remedy the invariance, we define the $2 \pi i$-periodic grand partition function by

$$
e^{J_{k}(\mu)}=\sum_{n=-\infty}^{\infty} e^{J_{k}^{\text {naive }}(\mu+2 \pi i n)}
$$

Here $J_{k}^{\text {naive }}(\mu)$ means the grand potential obtained from the naive small $k$, large $\mu$ asymptotic expansion, namely $J_{k}^{\text {naive }}(\mu)=J_{k}^{\text {(pert) }}(\mu)+J_{k}^{(\mathrm{np})}(\mu)$ with $J_{k}^{(\text {pert })}(\mu)$ given by (3.3). Through this periodic extension (3.8), the grand potential acquires the oscillatory part

$$
J_{k}(\mu)=J_{k}^{\text {naive }}(\mu)+J_{k}^{\text {osc }}(\mu),
$$

where $J_{k}^{\text {osc }}(\mu)$ is given by

$$
J_{k}^{\text {osc }}(\mu)=\log \left(1+\frac{1}{e^{J_{k}^{\text {naive }}(\mu)}} \sum_{n \neq 0} e^{J_{k}^{\text {naive }}(\mu+2 \pi i n)}\right) .
$$




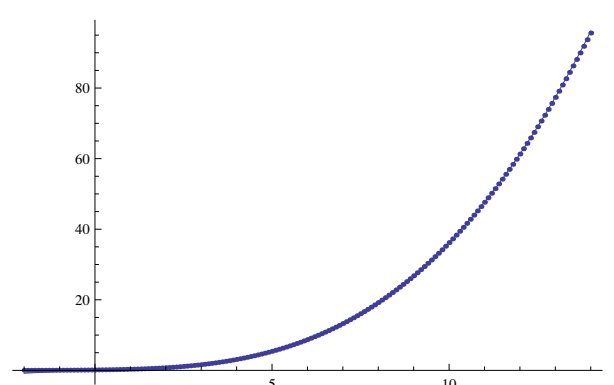

(a)

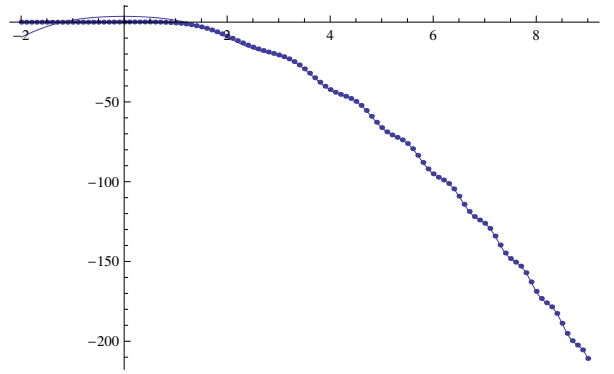

(c)

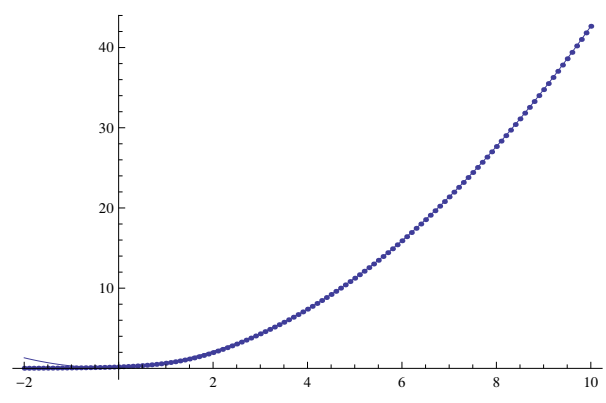

(b)

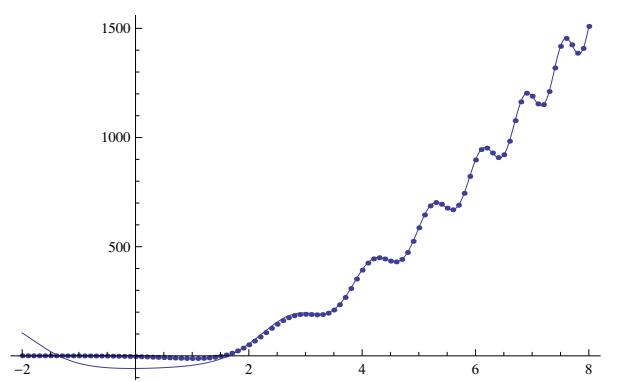

(d)

Figure 2. (a) The perturbative, (b) 1-instanton, (c) 2-instanton and (d) 3-instanton corrections of the grand potential $J_{k}(\mu)$ at $k=2$. The dots represent the grand potential obtained by the exact partition function while the solid lines represent the fitted functions.

For instance, the oscillatory term in the worldsheet 2-instanton comes from the $\pm 2 \pi i$ shift of the perturbative part $J_{k}^{(\text {pert })}(\mu)$. Since

$$
\frac{e^{J_{k}^{(\text {pert })}(\mu+2 \pi i)}}{e^{J_{k}^{(\text {pert })}(\mu)}}=\exp \left[-\frac{8 \mu}{k}+2 \pi i\left(C_{k} \mu^{2}+B_{k}-\frac{8}{3 k}\right)\right]
$$

we find the oscillatory term

$$
J_{k}^{\text {osc }}(\mu) \approx \frac{e^{J_{k}^{(\text {pert })}(\mu+2 \pi i)}+e^{J_{k}^{(\text {pert })}(\mu-2 \pi i)}}{e^{J_{k}^{(\text {pert })}(\mu)}}=2 \cos \left[2 \pi\left(C_{k} \mu^{2}+B_{k}-\frac{8}{3 k}\right)\right] e^{-\frac{8 \mu}{k}},
$$

in the worldsheet 2-instanton correction. Note that this exactly reproduces (3.6) at $k=1$.

We can proceed further to study the oscillatory term in the worldsheet 3-instanton for $k>2$ from the worldsheet 1-instanton contribution

$$
\begin{aligned}
J_{k}^{\mathrm{osc}}(\mu) & \approx \frac{e^{J_{k}^{(\mathrm{pert})}(\mu+2 \pi i)+J_{k}^{\mathrm{WS}(1)}(\mu+2 \pi i)}+e^{J_{k}^{(\mathrm{pert})}(\mu-2 \pi i)+J_{k}^{\mathrm{WS}(1)}(\mu-2 \pi i)}}{e^{J_{k}^{(\mathrm{pert})}(\mu)+J_{k}^{\mathrm{WS}(1)}(\mu)}} \\
& =2 \cos \left[2 \pi\left(C_{k} \mu^{2}+B_{k}-\frac{8}{3 k}\right)\right] e^{-\frac{8 \mu}{k}}+4 d_{k}^{(1)} \sin \frac{4 \pi}{k} \sin \left[2 \pi\left(C_{k} \mu^{2}+B_{k}-\frac{14}{3 k}\right)\right] e^{-\frac{12 \mu}{k}} .
\end{aligned}
$$

For $k=1,2$, however, since the D2 $m$-instanton $J_{k}^{\mathrm{D} 2(m)} \sim e^{-2 m \mu}(m=2,1$ for $k=1,2)$ has the same contribution as the worldsheet 1-instanton term $J_{k}^{\mathrm{WS}(1)}(\mu) \sim e^{-\frac{4 \mu}{k}}$, we have 


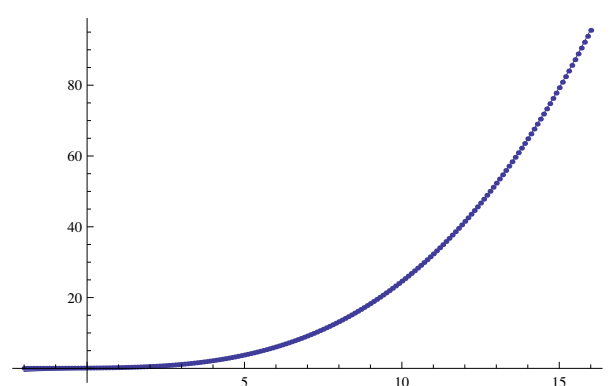

(a)

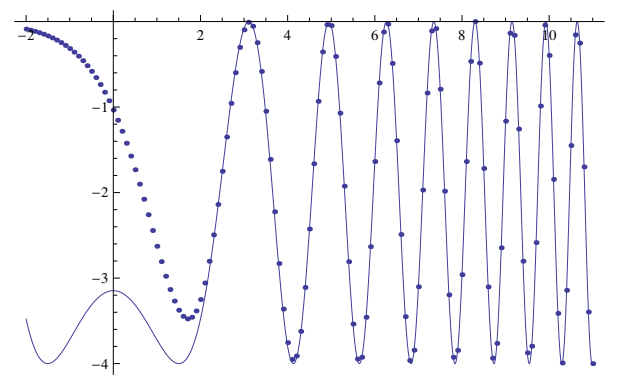

(c)

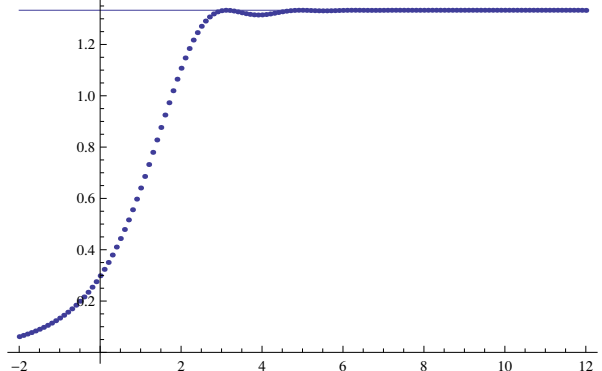

(b)

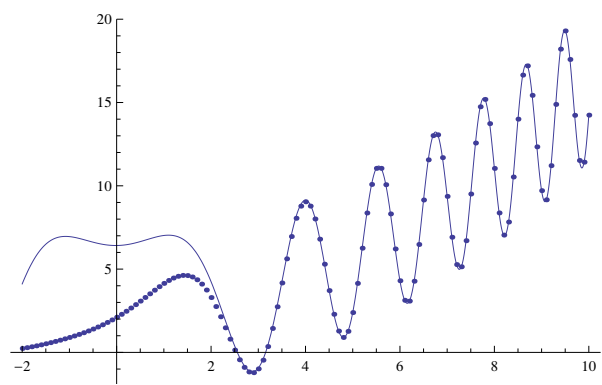

(d)

Figure 3. (a) The perturbative, (b) 1-instanton, (c) 2-instanton and (d) 3-instanton corrections of the grand potential $J_{k}(\mu)$ at $k=3$. The dots represent the grand potential obtained by the exact partition function while the solid lines represent the fitted functions.

to be careful. The mixing between these two kinds of instanton effects will be studied in more details in section 5. Using $\alpha_{k}^{(1)}$ and $\beta_{k}^{(1)}$ in (3.2), we find that the oscillatory term in the worldsheet 3 -instanton for $k=1,2$ is given by

$$
\begin{aligned}
J_{k}^{(3) \text { osc }}(\mu)= & -4 \pi\left(2 \alpha_{k}^{(1)} \mu+\beta_{k}^{(1)}\right) \sin \left[2 \pi\left(C_{k} \mu^{2}+B_{k}-\frac{8}{3 k}\right)\right] \\
& -8 \pi^{2} \alpha_{k}^{(1)} \cos \left[2 \pi\left(C_{k} \mu^{2}+B_{k}-\frac{8}{3 k}\right)\right] .
\end{aligned}
$$

Note that this expression for $k=1$ reproduces (3.7).

As we have seen in the previous subsection, we actually observed an oscillatory behavior in the numerical analysis of $J_{k}(\mu)$. Since the amplitude and the phase of oscillation perfectly matches (3.13) (and (3.14)), we strongly believe that our numerically observed oscillation is originated from the $2 \pi i$-periodic extension of the naive grand partition function.

One important consequence of the periodicity of the grand partition function is that in the integral transformation from $\Xi_{k}(\mu)$ to $Z_{k}(N)$ we can extend the integration range from $[-\pi i, \pi i]$ to $(-i \infty, i \infty)$

$$
Z_{k}(N)=\int_{-\pi i}^{\pi i} \frac{d \mu}{2 \pi i} e^{J_{k}(\mu)-N \mu}=\int_{-i \infty}^{i \infty} \frac{d \mu}{2 \pi i} e^{J_{k}^{\text {naive }}(\mu)-N \mu}
$$




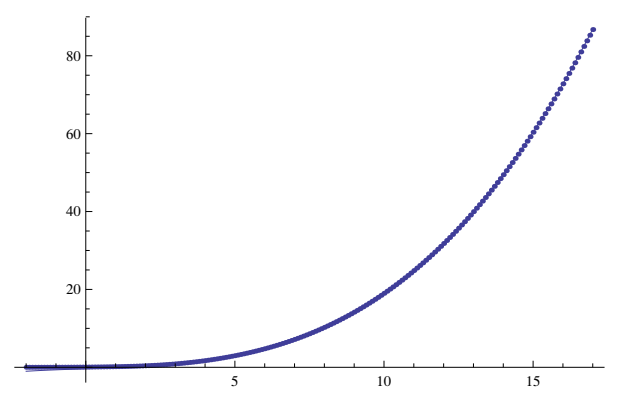

(a)

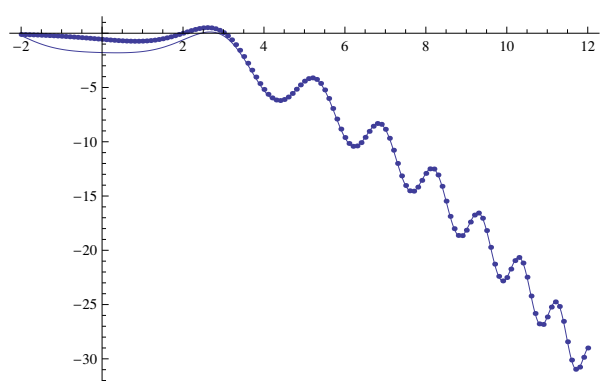

(c)

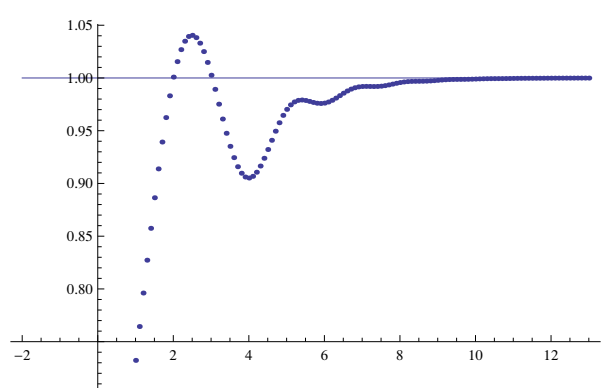

(b)

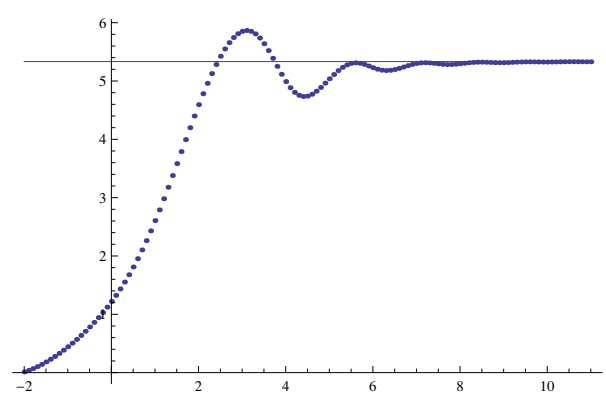

(d)

Figure 4. (a) The perturbative, (b) 1-instanton, (c) 2-instanton and (d) 3-instanton corrections of the grand potential $J_{k}(\mu)$ at $k=4$. The dots represent the grand potential obtained by the exact partition function while the solid lines represent the fitted functions.

Since the last expression of (3.15) does not involve $J_{k}^{\text {osc }}$, there is no oscillation in the canonical partition function $Z_{k}(N)$. The last expression in (3.15) also explains the good agreement of the Airy function with the exact partition function $Z_{k}(N){ }^{4}$

\subsection{Partition function}

In the previous subsections, we have studied the numerical behavior of the grand potential $J_{k}(\mu)$ and observed an interesting oscillating behavior. Though interesting from a theoretical viewpoint, the oscillating behavior causes a larger error in finding the exact values of instanton prefactors. As mentioned above, this behavior will disappear after an integral transformation from the grand potential to the canonical partition function. For this reason, as a complementary analysis, let us look at the partition function as well. We find exactly the same values from the fitting of $J_{k}(\mu)$ and the fitting of $Z_{k}(N)$. This agreement gives a strong support for the validity of our method.

Let us consider the case $k=1$. In this case, the non-perturbative correction to the grand potential starts from $\mathcal{O}\left(e^{-4 \mu}\right)$ as found in [13]. The (naive) grand potential is generically given by (3.2). We would like to determine the constants $\alpha_{1}^{(n)}, \beta_{1}^{(n)}$ and $\gamma_{1}^{(n)}$

\footnotetext{
${ }^{4}$ To obtain the Airy function expression, we need to deform the integration contour from $[-\pi i, \pi i]$ to $(-i \infty, i \infty)$. Naively, this deformation causes the error $\mathcal{O}\left(e^{-2 \mu_{*} / k}\right)=\mathcal{O}\left(e^{-\pi \sqrt{2 N / k}}\right)$, where $\mu_{*}$ is the value of the saddle-point, as was explained in [9]. However, such a correction does not actually appear in the difference of the exact partition function and the perturbative result. The procedure here naturally explains this fact.
} 


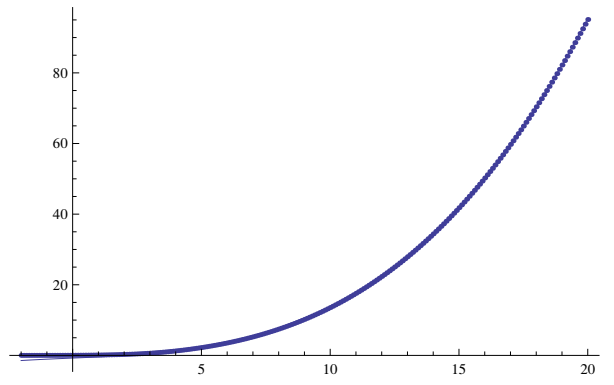

(a)

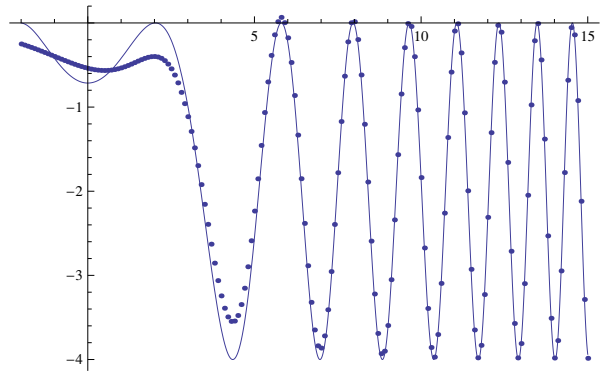

(c)

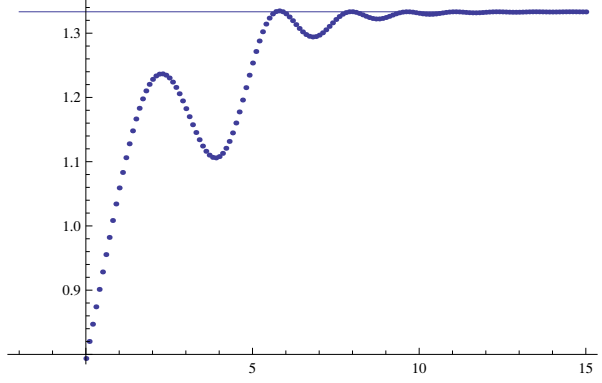

(b)

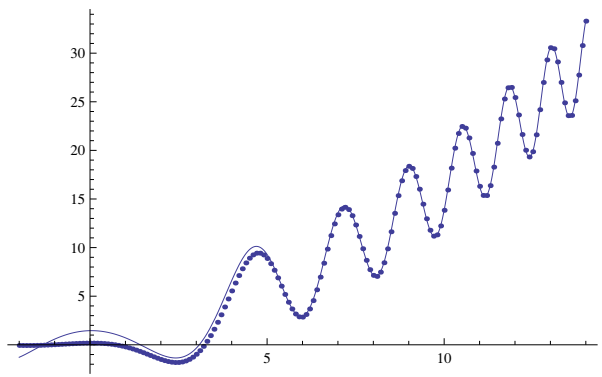

(d)

Figure 5. (a) The perturbative, (b) 1-instanton, (c) 2-instanton and (d) 3-instanton corrections of the grand potential $J_{k}(\mu)$ at $k=6$. The dots represent the grand potential obtained by the exact partition function while the solid lines represent the fitted functions.

from the exact data. Since the partition function is computed as

$$
\begin{aligned}
Z_{1}(N) & =\int_{-i \infty}^{i \infty} \frac{d \mu}{2 \pi i} e^{J_{1}^{\text {naive }}(\mu)-N \mu} \\
& =\int_{-i \infty}^{i \infty} \frac{d \mu}{2 \pi i} e^{J_{1}^{(\text {pert })}(\mu)-N \mu}\left[1+\left(\alpha_{1}^{(1)} \mu^{2}+\beta_{1}^{(1)} \mu+\gamma_{1}^{(1)}\right) e^{-4 \mu}+\cdots\right],
\end{aligned}
$$

the 1-instanton correction normalized by the perturbative partition function is given by

$$
\begin{aligned}
Z_{1}^{(1)}(N)= & \frac{1}{Z_{1}^{(\text {pert })}(N)} \int_{-i \infty}^{i \infty} \frac{d \mu}{2 \pi i} e^{J_{1}^{(\text {pert })}(\mu)-N \mu}\left(\alpha_{1}^{(1)} \mu^{2}+\beta_{1}^{(1)} \mu+\gamma_{1}^{(1)}\right) e^{-4 \mu} \\
= & \frac{\alpha_{1}^{(1)} \partial_{N}^{2}-\beta_{1}^{(1)} \partial_{N}+\gamma_{1}^{(1)}}{Z_{1}^{(\text {pert })}(N)} \int_{-i \infty}^{i \infty} \frac{d \mu}{2 \pi i} e^{J_{1}^{(\text {pert })}(\mu)-(N+4) \mu} \\
= & \left(C_{1}^{-1}\left(N+4-B_{1}\right) \alpha_{1}^{(1)}+\gamma_{1}^{(1)}\right) \frac{\operatorname{Ai}\left[C_{1}^{-1 / 3}\left(N+4-B_{1}\right)\right]}{\operatorname{Ai}\left[C_{1}^{-1 / 3}\left(N-B_{1}\right)\right]} \\
& -C_{1}^{-1 / 3} \beta_{1}^{(1)} \frac{\operatorname{Ai}^{\prime}\left[C_{1}^{-1 / 3}\left(N+4-B_{1}\right)\right]}{\operatorname{Ai}\left[C_{1}^{-1 / 3}\left(N-B_{1}\right)\right]}
\end{aligned}
$$

where $Z_{k}^{(\text {pert })}(N)$ is the perturbative contribution, which is given by [8,9],

$$
Z_{k}^{(\text {pert })}(N)=C_{k}^{-1 / 3} e^{A_{k}} \mathrm{Ai}\left[C_{k}^{-1 / 3}\left(N-B_{k}\right)\right] .
$$




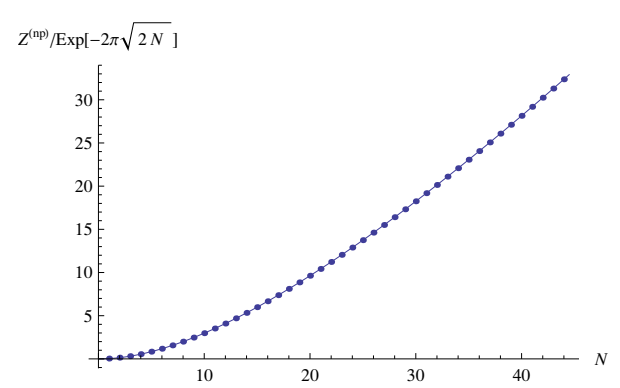

(a)

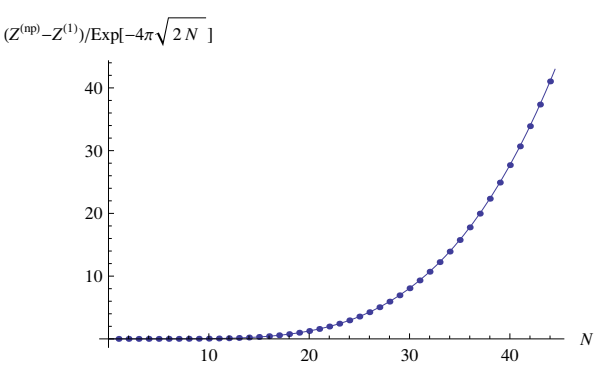

(b)

Figure 6. The 1- and 2-instanton corrections to the partition function at $k=1$. We plot (a) $Z_{1}^{(\mathrm{np})} / e^{-2 \pi \sqrt{2 N}}$ and (b) $\left(Z_{1}^{(\mathrm{np})}-Z_{1}^{(1)}\right) / e^{-4 \pi \sqrt{2 N}}$ against $N$. The instanton corrections in terms of the Airy function (the solid lines) excellently agree with the exact values (the dots) even for small $N$.

The expression (3.17) is the form expected from the Fermi gas consideration. We compare this result with the non-perturbative correction computed from the exact values of the partition function using our definition of $Z_{1}^{(\mathrm{np})}(N)$ in (1.6). To fix the constants $\alpha_{1}^{(1)}, \beta_{1}^{(1)}$ and $\gamma_{1}^{(1)}$, we fit the exact data $Z_{1}^{(\mathrm{np})}(N) / e^{-2 \pi \sqrt{2 N}}$ for $20 \leq N \leq 44$ by the expected form $Z_{1}^{(1)}(N) / e^{-2 \pi \sqrt{2 N}}$, and find that $\alpha_{1}^{(1)}, \beta_{1}^{(1)}$ and $\gamma_{1}^{(1)}$ are very close to (in about 14-digit agreement)

$$
\alpha_{1}^{(1)}=\frac{4}{\pi^{2}}, \quad \beta_{1}^{(1)}=\frac{1}{\pi^{2}}, \quad \gamma_{1}^{(1)}=\frac{1}{4 \pi^{2}} .
$$

This result, of course, is consistent with (3.5) obtained from the fitting of $J_{1}(\mu)$. In figure 6 (a), we show the exact data $Z_{1}^{(\mathrm{np})}(N) / e^{-2 \pi \sqrt{2 N}}$ (the $\operatorname{dots}$ ) and our result $Z_{1}^{(1)}(N) / e^{-2 \pi \sqrt{2 N}}$ with (3.19) (the solid line). Our Airy function expression of the instanton correction (3.17) is in excellent agreement with the exact data from $N=1$ to $N=44$. In the large $N$ limit, $Z_{1}^{(1)}(N)$ is asymptotically expanded as

$$
\begin{aligned}
Z_{1}^{(1)}(N)= & e^{-2 \pi \sqrt{2 N}}\left[2 N-\frac{13 \pi^{2}-2}{2 \sqrt{2} \pi} \sqrt{N}+\frac{169 \pi^{4}+116 \pi^{2}+8}{32 \pi^{2}}\right. \\
& \left.-\frac{\pi\left(2197 \pi^{2}+4012\right)}{384 \sqrt{2 N}}+\frac{28561 \pi^{4}+73424 \pi^{2}-32768}{12288 N}+\mathcal{O}\left(N^{-3 / 2}\right)\right] .
\end{aligned}
$$

The leading term $2 N e^{-2 \pi \sqrt{2 N}}$ agrees with the result in [14]. Note that our result is exact at all orders in $1 / N$.

Once we fix $\alpha_{1}^{(1)}, \beta_{1}^{(1)}$ and $\gamma_{1}^{(1)}$, we can proceed to determining $\alpha_{1}^{(2)}, \beta_{1}^{(2)}$ and $\gamma_{1}^{(2)}$. The 2-instanton correction to the partition function is given by

$$
\begin{aligned}
Z_{1}^{(2)}(N)= & \left(2 N^{2}+\frac{2 \pi^{4} \alpha_{1}^{(2)} C_{1}+3 C_{1}+244}{8} N\right. \\
& \left.+\frac{32 \pi^{4} \gamma_{1}^{(2)}+1}{128} C_{1}^{2}+\frac{122 \pi^{4} \alpha_{1}^{(2)}+119}{64} C_{1}+\frac{3721}{32}\right) \frac{\mathrm{Ai}\left[C_{1}^{-1 / 3}\left(N+8-B_{1}\right)\right]}{\operatorname{Ai}\left[C_{1}^{-1 / 3}\left(N-B_{1}\right)\right]} \\
& -C_{1}^{2 / 3}\left(N+\frac{4 \pi^{4} \beta_{1}^{(2)}+1}{16} C_{1}+\frac{29}{8}\right) \frac{\operatorname{Ai}^{\prime}\left[C_{1}^{-1 / 3}\left(N+8-B_{1}\right)\right]}{\operatorname{Ai}\left[C_{1}^{-1 / 3}\left(N-B_{1}\right)\right]}
\end{aligned}
$$


In the same way as above, from the numerical fitting we find

$$
\alpha_{1}^{(2)}=-\frac{26}{\pi^{2}}, \quad \beta_{1}^{(2)}=-\frac{1}{4 \pi^{2}}, \quad \gamma_{1}^{(2)}=-\frac{9}{32 \pi^{2}}+2 .
$$

These are in about 14-digit agreement with the fitted values. In figure 6(b), we show the exact data $\left(Z_{1}^{(\mathrm{np})}(N)-Z_{1}^{(1)}(N)\right) / e^{-4 \pi \sqrt{2 N}}$ and our result $Z_{1}^{(2)}(N) / e^{-4 \pi \sqrt{2 N}}$ with (3.22).

Repeating this method, we find the values of $\alpha_{1}^{(n)}, \beta_{1}^{(n)}$ and $\gamma_{1}^{(n)}$ up to $n=4$. Furthermore we can apply this procedure to other levels. Our results at $k=1,2,3,4,6$ are summarized in (1.5).

Using (1.5), one can easily know the instanton corrections to the partition function. We have indeed checked that the instanton corrections to the partition function obtained from (1.5) precisely agree with the exact data. The results in (1.5) show that the prefactors at $k=2,6$ are strongly related to those at $k=1,3$, respectively. Currently, we do not understand the physical origin of this relation. It would be interesting to study what this means.

\section{Worldsheet instantons}

In the previous section, we have obtained the exact non-perturbative correction from the numerical study of the grand potential $J_{k}(\mu)$ and the partition function $Z_{k}(N)$. The nonperturbative correction consists of several contributions written as (1.1). In this section, we would like to determine the prefactors of worldsheet instanton corrections. The prefactors of the D2-instanton corrections will be considered in section 5. The prefactor $d_{k}^{(n)}$ of the worldsheet $n$-instanton is expected to be independent of $\mu$. We can determine $d_{k}^{(n)}$ from the topological string on local $\mathbb{F}_{0}$ (note that $\mathbb{F}_{0} \cong \mathbb{P}^{1} \times \mathbb{P}^{1}$ ).

\subsection{Topological string on local $\mathbb{F}_{0}$}

As discussed in $[6,20]$, the ABJM matrix model (2.1) (and its generalization to the ABJ model [21]) is related to the lens space matrix model $[22,23]^{5}$ by an analytic continuation of the rank of the gauge group, and it is further related to the topological string on local $\mathbb{F}_{0}$ via a large $N$ duality. Using this chain of dualities, we can compute the worldsheet instanton corrections in the ABJM theory from the knowledge of the topological string on local $\mathbb{F}_{0}$.

In general, the worldsheet instanton sum in the topological string has the following structure [25]

$$
F^{\mathrm{top}}=\sum_{n=1}^{\infty} \sum_{g, \vec{d}} n_{\vec{d}}^{g}\left(2 \sin \frac{n \lambda_{s}}{2}\right)^{2 g-2} \frac{e^{-n \vec{d} \cdot \vec{T}}}{n},
$$

where $\lambda_{s}$ denotes the string coupling of topological string theory.

The free energy of ABJM matrix model and the free energy of topological string on local $\mathbb{F}_{0}$ are related by a Fourier transformation [9] due to the wavefunction nature of the

\footnotetext{
${ }^{5}$ The ABJ(M) matrix model and the lens space matrix model are also closely related to the supermatrix model in [24].
} 
topological string partition functions [26]. Since the canonical partition function $Z_{k}(N)$ is obtained from the integral transform of grand partition function $e^{J_{k}(\mu)}$, it is natural to identify the grand potential $J_{k}(\mu)$ with the free energy $F^{\text {top }}\left(T_{1}, T_{2}\right)$ of topological string on local $\mathbb{F}_{0}$. Here $T_{1}$ and $T_{2}$ denote the Kähler parameters of the two $\mathbb{P}^{1}$ 's in $\mathbb{F}_{0}$. Since the ABJM slice is defined by $T_{1}=T_{2}$, we arrive at the relation

$$
J_{k}(\mu)=F^{\text {top }}\left(T_{1}=T_{2} \equiv T\right),
$$

with $T$ and $\mu$ related by [9]

$$
T=\frac{4 \mu}{k}-\pi i .
$$

To compare the topological string and ABJM theory, we should be careful about the normalization of string coupling. The string coupling $\lambda_{s}$ in the topological string theory and the string coupling $g_{s}=\frac{2 \pi i}{k}$ in the ABJM theory are related by

$$
g_{s}^{2 g-2} \widetilde{F}_{g}^{\text {top }}=\lambda_{s}^{2 g-2} F_{g}^{\text {top }}
$$

where [9]

$$
\widetilde{F}_{g}^{\text {top }}=(-4)^{g-1} F_{g}^{\text {top }}, \quad(g \geq 1)
$$

Therefore, $\lambda_{s}$ is given by

$$
\lambda_{s}=-2 i g_{s}=\frac{4 \pi}{k},
$$

and the worldsheet instanton sum (4.1) becomes

$$
F^{\mathrm{top}}=\sum_{n=1}^{\infty} \sum_{g, \vec{d}} n_{\vec{d}}^{g}\left(2 \sin \frac{2 \pi n}{k}\right)^{2 g-2} \frac{e^{-n \vec{d} \cdot \vec{T}}}{n} .
$$

The Gopakumar-Vafa invariants $n_{\vec{d}}^{g}$ of local $\mathbb{F}_{0}$ were computed in [28]. Up to total degree $d_{1}+d_{2}=3$, the GV invariants $n_{\vec{d}}^{g}$ are given by

$$
n_{1,0}^{0}=n_{0,1}^{0}=-2, \quad n_{1,1}^{0}=-4, \quad n_{2,1}^{0}=n_{1,2}^{0}=-6,
$$

and $n_{\vec{d}}^{g}=0(g \geq 1)$ for the above cases. Hence $F^{\text {top }}$ of local $\mathbb{F}_{0}$ is expanded as

$$
F^{\mathrm{top}}=-\sum_{n=1}^{\infty}\left[\frac{e^{-n T_{1}}+e^{-n T_{2}}}{2 n \sin ^{2} \frac{2 \pi n}{k}}+\frac{e^{-n\left(T_{1}+T_{2}\right)}}{n \sin ^{2} \frac{2 \pi n}{k}}+\frac{3\left(e^{-n\left(2 T_{1}+T_{2}\right)}+e^{-n\left(T_{1}+2 T_{2}\right)}\right)}{2 n \sin ^{2} \frac{2 \pi n}{k}}+\cdots\right]
$$

Using the relations (4.2) and (4.3), we can read off the coefficient $d_{k}^{(n)}$ of the worldsheet $n$-instanton $e^{-\frac{4 n \mu}{k}}$. The first three coefficients are given by the first line of (1.3). More generally, we can write down the worldsheet instanton correction to the grand potential in the form

$$
J_{k}^{\mathrm{WS}}(\mu)=\sum_{n, g, d=1}^{\infty} n_{d}^{g}\left(2 \sin \frac{2 \pi n}{k}\right)^{2 g-2} \frac{(-1)^{d n} e^{-\frac{4 d n \mu}{k}}}{n}
$$


where $n_{d}^{g}$ denotes the weighted sum of GV invariants on local $\mathbb{F}_{0}[27]$

$$
n_{d}^{g}=\sum_{d_{1}+d_{2}=d} n_{d_{1}, d_{2}}^{g} .
$$

For instance, using the data $n_{4}^{0}=-48, n_{4}^{1}=9, n_{4}^{g}=0(g \geq 2)$ and (4.8), we find the 4 -instanton coefficient $d_{k}^{(4)}$ in (1.3). In a similar manner, we can determine the higher instanton coefficients using the data of GV invariants in $[27,28]$.

\subsection{Worldsheet instanton in the 't Hooft limit}

In the previous subsection we have determined the worldsheet instanton correction $J_{k}^{\mathrm{WS}}(\mu)$ from the topological string on local $\mathbb{F}_{0}$. As a consistency check, we consider the 't Hooft limit of the worldsheet instanton corrections derived from the results in the previous subsection and compare with the known results of the genus-zero and genus-one free energy. In the 't Hooft limit, the free energy has the genus expansion

$$
F\left(g_{s}, \lambda\right) \equiv \log Z\left(g_{s}, \lambda\right)=\sum_{g=0}^{\infty} g_{s}^{2 g-2} F_{g}(\lambda)
$$

where $g_{s}=\frac{2 \pi i}{k}$ is the string coupling and $\lambda \equiv \frac{N}{k}$ is the 't Hooft coupling.

One easily finds that the worldsheet instanton corrections to the partition function up to 3-instanton are given by ${ }^{6}$

$$
\begin{aligned}
& Z_{k}^{\mathrm{WS}(1)}(N)=d_{k}^{(1)} \frac{\operatorname{Ai}\left[C_{k}^{-1 / 3}\left(N+\frac{4}{k}-B_{k}\right)\right]}{\operatorname{Ai}\left[C_{k}^{-1 / 3}\left(N-B_{k}\right)\right]} \\
& Z_{k}^{\mathrm{WS}(2)}(N)=\left(d_{k}^{(2)}+\frac{\left(d_{k}^{(1)}\right)^{2}}{2}\right) \frac{\operatorname{Ai}\left[C_{k}^{-1 / 3}\left(N+\frac{8}{k}-B_{k}\right)\right]}{\operatorname{Ai}\left[C_{k}^{-1 / 3}\left(N-B_{k}\right)\right]} \\
& Z_{k}^{\mathrm{WS}(3)}(N)=\left(d_{k}^{(3)}+d_{k}^{(2)} d_{k}^{(1)}+\frac{\left(d_{k}^{(1)}\right)^{3}}{6}\right) \frac{\operatorname{Ai}\left[C_{k}^{-1 / 3}\left(N+\frac{12}{k}-B_{k}\right)\right]}{\operatorname{Ai}\left[C_{k}^{-1 / 3}\left(N-B_{k}\right)\right]},
\end{aligned}
$$

where $d_{k}^{(n)}(n=1,2,3)$ are given by (1.3). It is convenient to introduce $\hat{N}=N-\frac{k}{24}$ in the analysis below. In the large $\hat{N}$ limit, ${ }^{7} Z_{k}^{\mathrm{WS}(1)}(N)$ is asymptotically expanded as

$$
Z_{k}^{\mathrm{WS}(1)}(N)=\frac{e^{-2 \pi \sqrt{2 \hat{N} / k}}}{\sin ^{2}(2 \pi / k)}\left[1-\frac{5 \sqrt{2} \pi}{3 k^{2}} \sqrt{\frac{k}{\hat{N}}}+\left(\frac{25 \pi^{2}}{9 k^{4}}-\frac{1}{k^{2}}\right) \frac{k}{\hat{N}}+\mathcal{O}\left(\left(\frac{k}{\hat{N}}\right)^{3 / 2}\right)\right] .
$$

If we further expand this in $g_{s}=\frac{2 \pi i}{k}$, we obtain

$$
Z_{k}^{\mathrm{WS}(1)}(N)=e^{-2 \pi \sqrt{2 \hat{\lambda}}}\left[-g_{s}^{-2}+\frac{1}{3}-\frac{5}{6 \sqrt{2} \pi \sqrt{\hat{\lambda}}}-\frac{1}{4 \pi^{2} \hat{\lambda}}+\mathcal{O}\left(g_{s}^{2}\right)\right]
$$

\footnotetext{
${ }^{6}$ We assume that the worldsheet instanton corrections up to 3-instanton are not mixed to the D2instanton corrections. This assumption is true for $k>6$, and the 't Hooft limit, of course, satisfies this condition.

${ }^{7}$ Strictly, we consider the large $\hat{\lambda}=\hat{N} / k$ limit (strong coupling limit).
} 
where $\hat{\lambda} \equiv \frac{\hat{N}}{k}=\lambda-\frac{1}{24}$. Thus the worldsheet 1-instanton correction to the free energy are given by

$$
F_{g=0}^{\mathrm{WS}(1)}(\hat{\lambda})=-e^{-2 \pi \sqrt{2 \hat{\lambda}}}, \quad F_{g=1}^{\mathrm{WS}(1)}(\hat{\lambda})=\left[\frac{1}{3}-\frac{5}{6 \sqrt{2} \pi \sqrt{\hat{\lambda}}}-\frac{1}{4 \pi^{2} \hat{\lambda}}\right] e^{-2 \pi \sqrt{2 \hat{\lambda}}} .
$$

In a similar way, we obtain the genus-zero and genus-one part of the 2- and 3-instanton corrections from (4.13) as

$$
\begin{aligned}
& F_{g=0}^{\mathrm{WS}(2)}(\hat{\lambda})=\left[\frac{9}{8}+\frac{1}{\pi \sqrt{2 \hat{\lambda}}}\right] e^{-4 \pi \sqrt{2 \hat{\lambda}}} \\
& F_{g=1}^{\mathrm{WS}(2)}(\hat{\lambda})=\left[-\frac{1}{2}+\frac{83}{24 \sqrt{2} \pi \sqrt{\hat{\lambda}}}+\frac{91}{48 \pi^{2} \hat{\lambda}}+\frac{23}{24 \sqrt{2} \pi^{3} \hat{\lambda}^{3 / 2}}+\frac{1}{8 \pi^{4} \hat{\lambda}^{2}}\right] e^{-4 \pi \sqrt{2 \hat{\lambda}}}
\end{aligned}
$$

and

$$
\begin{aligned}
F_{g=0}^{\mathrm{WS}(3)}(\hat{\lambda})= & {\left[-\frac{82}{27}-\frac{9}{2 \sqrt{2} \pi \sqrt{\hat{\lambda}}}-\frac{1}{\pi^{2} \hat{\lambda}}-\frac{1}{6 \sqrt{2} \pi^{3} \hat{\lambda}^{3 / 2}}\right] e^{-6 \pi \sqrt{2 \hat{\lambda}}}, } \\
F_{g=1}^{\mathrm{WS}(3)}(\hat{\lambda})= & {\left[\frac{10}{9}-\frac{1205}{54 \sqrt{2} \pi \sqrt{\hat{\lambda}}}-\frac{1145}{72 \pi^{2} \hat{\lambda}}-\frac{185}{16 \sqrt{2} \pi^{3} \hat{\lambda}^{3 / 2}}\right.} \\
& \left.-\frac{47}{16 \pi^{4} \hat{\lambda}^{2}}-\frac{47}{48 \sqrt{2} \pi^{5} \hat{\lambda}^{5 / 2}}-\frac{1}{12 \pi^{6} \hat{\lambda}^{3}}\right] e^{-6 \pi \sqrt{2 \hat{\lambda}}},
\end{aligned}
$$

where we have used the relation between the instanton corrections to the partition function and to the free energy:

$$
\begin{aligned}
& F_{k}^{\mathrm{WS}(1)}(N)=Z_{k}^{\mathrm{WS}(1)}(N), \quad F_{k}^{\mathrm{WS}(2)}(N)=Z_{k}^{\mathrm{WS}(2)}(N)-\frac{1}{2}\left(Z_{k}^{\mathrm{WS}(1)}(N)\right)^{2}, \\
& F_{k}^{\mathrm{WS}(3)}(N)=Z_{k}^{\mathrm{WS}(3)}(N)-Z_{k}^{\mathrm{WS}(2)}(N) Z_{k}^{\mathrm{WS}(1)}(N)+\frac{1}{3}\left(Z_{k}^{\mathrm{WS}(1)}(N)\right)^{3} .
\end{aligned}
$$

Let us compare these results with the result in [6]. One immediately finds that the instanton corrections to the genus-zero free energy exactly agree with the result in [6] (see also [19]). Let us consider the genus-one free energy. The genus-one free energy is given by

$$
F_{g=1}=-\log \eta(\tau)
$$

where $\eta(\tau)$ is the Dedekind eta function, and the modulus $\tau$ is related to the 't Hooft coupling through

$$
\tau=i \frac{K^{\prime}\left(\frac{i \kappa}{4}\right)}{K\left(\frac{i \kappa}{4}\right)}, \quad \lambda=\frac{\kappa}{8 \pi}{ }_{3} F_{2}\left(\frac{1}{2}, \frac{1}{2}, \frac{1}{2} ; 1, \frac{3}{2} ;-\frac{\kappa^{2}}{16}\right) .
$$

In the strong coupling limit, the genus-one free energy behaves as

$$
F_{g=1}=\frac{\pi \sqrt{2 \hat{\lambda}}}{6}-\frac{1}{2} \log (2 \sqrt{2 \hat{\lambda}})+\left[\frac{1}{3}-\frac{5}{6 \sqrt{2} \pi \sqrt{\hat{\lambda}}}-\frac{1}{4 \pi^{2} \hat{\lambda}}\right] e^{-2 \pi \sqrt{2 \hat{\lambda}}}+\mathcal{O}\left(e^{-4 \pi \sqrt{2 \hat{\lambda}}}\right) .
$$




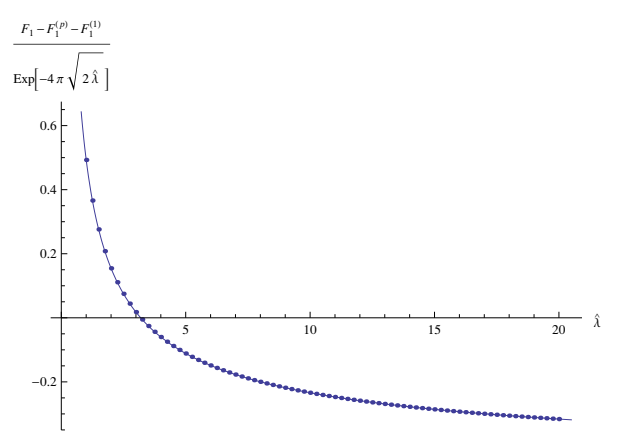

(a)

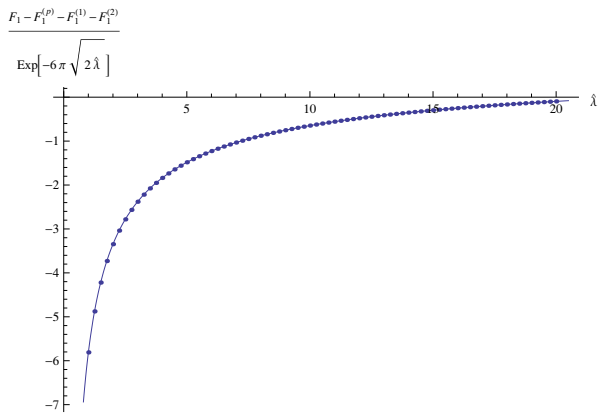

(b)

Figure 7. (a) The 2- and (b) 3-instanton corrections to the genus one free energy.

The 1-instanton correction exactly agrees with (4.16). It becomes messy to compute the higher instanton corrections. Instead, we numerically evaluate (4.20), and extract the 2- and 3-instanton corrections from it. In figure $7(\mathrm{a})$, we plot $\left(F_{g=1}-F_{g=1}^{\text {(pert) }}-\right.$ $\left.F_{g=1}^{\mathrm{WS}(1)}\right) / e^{-4 \pi \sqrt{2 \hat{\lambda}}}$ against $\hat{\lambda}$. The dots represent the numerical data obtained from (4.20) and (4.22) while the solid line represents our prediction (4.17). Our results show a perfect agreement with the numerical evaluation. Similarly, in figure $7(\mathrm{~b})$, we plot $\left(F_{g=1}-F_{g=1}^{\text {(pert) }}-\right.$ $\left.F_{g=1}^{\mathrm{WS}(1)}-F_{g=1}^{\mathrm{WS}(2)}\right) / e^{-6 \pi \sqrt{2 \hat{\lambda}}}$ against $\hat{\lambda}$. Our 3-instanton prediction (4.18) again agrees with the numerical ones.

\section{Conjecture of D2-instanton corrections}

The coefficients $d_{k}^{(n)}$ of the worldsheet $n$-instanton corrections diverge when $k \in 2 n / \mathbb{N}$. However, we confirmed numerically that the grand potential itself is finite at these values of $k$. Furthermore, at $k=2 n / m(m=1,2, \ldots)$, the worldsheet $n$-instanton correction is just the same order as the D2 $m$-instanton correction because $e^{-4 n \mu / k}=e^{-2 m \mu}$. Therefore it is natural to expect that D2-instanton corrections are also divergent at these values of $k$, and the divergence coming from the worldsheet instanton is canceled by the divergence from D2-instanton. The sum of these two contributions leads to a finite result. This story imposes a strong constraint on the pole structure of the D2-instanton corrections.

\subsection{D2 1-instanton correction}

Let us consider the $e^{-2 \mu}$ term in $J_{k}^{(\mathrm{np})}(\mu)$. At $k=2 n$ with $n \in \mathbb{Z}$, the $e^{-2 \mu}$ term receives two contributions: one from the D2 1-instanton and the other from worldsheet $n$-instanton. The coefficient of worldsheet $n$-instanton is divergent in the limit $k \rightarrow 2 n$. This divergence has the general structure

$$
\lim _{k \rightarrow 2 n} d_{k}^{(n)} e^{-\frac{4 n \mu}{k}}=(-1)^{n-1}\left[\frac{4 n}{\pi^{2}(k-2 n)^{2}}+\frac{4(\mu+1)}{\pi^{2}(k-2 n)}+\frac{2 \mu^{2}+2 \mu+1}{n \pi^{2}}+w^{(n)}\right] e^{-2 \mu},
$$


with some constant $w^{(n)}$. We have checked this behavior up to $n=7$ using the GopakumarVafa invariant of local $\mathbb{F}_{0}$ in $[28]$ and found

$$
\begin{aligned}
w^{(1)} & =\frac{1}{3}, & w^{(2)} & =\frac{7}{6}, \quad w^{(3)}=\frac{37}{9}, \quad w^{(4)}=\frac{187}{12}, \quad w^{(5)}=\frac{661}{15}+12 \sqrt{5}, \\
w^{(6)} & =\frac{6919}{18}, & w^{(7)} & =\frac{19783-16380 \cos \frac{\pi}{7}+29903 \cos \frac{2 \pi}{7}-22428 \cos \frac{3 \pi}{7}}{42 \sin ^{2} \frac{\pi}{7}} .
\end{aligned}
$$

Next, let us consider the D2 1-instanton correction

$$
J_{k}^{\mathrm{D} 2(1)}=\left[a_{k}^{(1)} \mu^{2}+b_{k}^{(1)} \mu+c_{k}^{(1)}\right] e^{-2 \mu} .
$$

The small $k$ expansion of the coefficients $a_{k}^{(1)}, b_{k}^{(1)}$ and $c_{k}^{(1)}$ was computed in [9] up to $k^{3}$ :

$$
\begin{aligned}
& a_{k}^{(1)}=-\frac{4}{\pi^{2} k}+\frac{k}{2}-\frac{\pi^{2} k^{3}}{96}+\cdots, \\
& b_{k}^{(1)}=\frac{4}{\pi^{2} k}-\frac{5 k}{6}+\frac{67 \pi^{2} k^{3}}{1440}+\cdots, \\
& c_{k}^{(1)}=\frac{4}{\pi^{2} k}-\frac{2}{3 k}+\frac{\pi^{2}-1}{12} k-\frac{\pi^{2}\left(104+5 \pi^{2}\right)}{2880} k^{3}+\cdots .
\end{aligned}
$$

From our results at $k=1,3$, we expect that the coefficients of $e^{-2 \mu}$ vanish for odd $k \in \mathbb{Z}$. $^{8}$ Also, in order to cancel the poles coming from the worldsheet instanton (5.1), the D2 1-instanton coefficients should behave as

$$
\begin{aligned}
\lim _{k \rightarrow 2 n} a_{k}^{(1)} & =\mathcal{O}(1), \\
\lim _{k \rightarrow 2 n} b_{k}^{(1)} & =-\frac{4(-1)^{n-1}}{\pi^{2}(k-2 n)}+\mathcal{O}(1), \\
\lim _{k \rightarrow 2 n} c_{k}^{(1)} & =(-1)^{n-1}\left[-\frac{4 n}{\pi^{2}(k-2 n)^{2}}-\frac{4}{\pi^{2}(k-2 n)}\right]+\mathcal{O}(1) .
\end{aligned}
$$

From the above conditions, we can fix the forms of $a_{k}^{(1)}, b_{k}^{(1)}$ and $c_{k}^{(1)}$. The result is given by (1.4). Summing the contributions from the worldsheet $n$-instanton and the D2 1instanton, the $e^{-2 \mu}$ term at $k=2 n$ gives the finite answer

$$
J_{2 n}^{\mathrm{D} 2(1)}(\mu)+J_{2 n}^{\mathrm{WS}(n)}(\mu)=(-1)^{n-1}\left[\frac{4 \mu^{2}+2 \mu+1}{n \pi^{2}}+s^{(n)}\right] e^{-2 \mu},
$$

where

$$
s^{(n)}=w^{(n)}+\frac{1}{3 n}-\frac{2 n}{3} .
$$

For lower $n$, we find

$$
s^{(1)}=0, \quad s^{(2)}=0, \quad s^{(3)}=\frac{20}{9}, \quad s^{(4)}=13,
$$

${ }^{8}$ For odd $k$, it is plausible that there are no D2-branes wrapping $\mathbb{R P}^{3}$ as in the case of $k=1$ discussed in [14]. Thus it is expected that the D2-instanton correction starts from $\mathcal{O}\left(e^{-2 \pi \sqrt{2 k N}}\right)$. Further, we have numerically checked that this expectation is true at $k=5,7$ by using the TBA-like equations in [13]. 


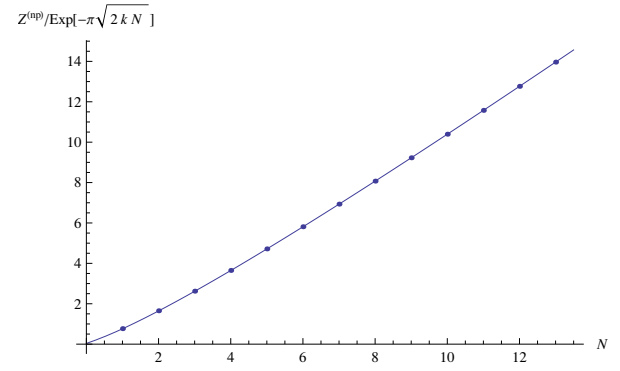

(a)

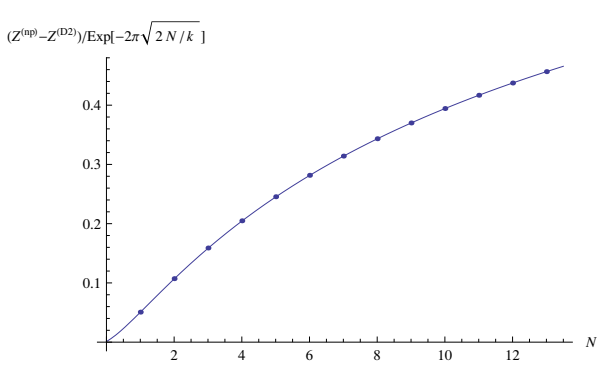

(b)

Figure 8. In these figures, we plot (a) the leading and (b) the next-to-leading contributions of the instanton corrections at $k=\frac{3}{2}$. The dots represent the numerical values from the TBA-like equations while the solid lines represent our theoretical predictions. The leading correction is captured by the D2 1-instanton, and the next-to-leading correction by the worldsheet 1-instanton.

and (5.6) for $n=1,2,3$ correctly reproduce our results at $k=2,4,6$ in (1.5)!

As a further check of our conjecture (1.4), we compare the instanton corrections to the partition function at various $k$ with the numerical results obtained by using the TBA-like equations. As explained in [13] (see also [14]), the ABJM partition function can be also computed by solving the TBA-type integral equations. ${ }^{9}$ In figure 8 , for instance, we show the instanton corrections at $k=\frac{3}{2}$. At $k=\frac{3}{2}$, the leading contribution is the D2 1-instanton correction. In figure $8(\mathrm{a})$, the dots represent $Z_{3 / 2}^{(\mathrm{np})}(N) / e^{-\pi \sqrt{2 k N}}$ computed by the TBA-like equations while the solid line represents the D2 1-instanton correction $Z_{3 / 2}^{\mathrm{D} 2}(N) / e^{-\pi \sqrt{2 k N}}$ given by (1.7) with our conjecture (1.4). In figure 8(b), we plot the next-to-leading contribution. At this order, the worldsheet 1-instanton correction is dominant. The dots represent $\left(Z_{3 / 2}^{(\mathrm{np})}(N)-Z_{3 / 2}^{\mathrm{D} 2}(N)\right) / e^{-2 \pi \sqrt{2 N / k}}$ computed by the TBA-like equations (and (1.7)) while the solid line represents the worldsheet 1-instanton correction $Z_{3 / 2}^{\mathrm{WS}}(N) / e^{-2 \pi \sqrt{2 N / k}}$ given by (1.8). In both figures, our theoretical predictions (1.7) and (1.8) perfectly explain the numerical results from TBA. We have checked the validity of our conjecture (1.4) for other $k$ 's in the same way.

\subsection{Comment on D2 2-instanton correction}

By the similar reasoning, it seems to be possible to guess the forms of D2 2-instanton coefficients $a_{k}^{(2)}, b_{k}^{(2)}$ and $c_{k}^{(2)}$. However, the constraint is much weaker than the 1-instanton case, hence we can not determine the forms of 2-instanton coefficients completely. We need more information to fix them.

\footnotetext{
${ }^{9}$ One advantage to use the TBA-like equations is that we can numerically compute the partition function at any $k$. However, we cannot neglect numerical errors as $N$ grows.
} 
The $k \rightarrow n$ limit of worldsheet $n$-instanton has the general structure

$$
\lim _{k \rightarrow n} d_{k}^{(n)} e^{-\frac{4 n \mu}{k}}=-\left(4+5(-1)^{n}\right)\left[\frac{n}{4 \pi^{2}(k-n)^{2}}+\frac{2 \mu+1}{2 \pi^{2}(k-n)}+\frac{2 \mu^{2}+\mu+\frac{1}{4}}{n \pi^{2}}+v^{(n)}\right] e^{-4 \mu} .
$$

We have checked this behavior up to $n=7$ and found

$$
\begin{array}{ll}
v^{(1)}=\frac{1}{3}, & v^{(2)}=\frac{1}{18}, \quad v^{(3)}=\frac{37}{9}, \quad v^{(4)}=\frac{13}{36}, \quad v^{(5)}=\frac{661}{15}-12 \sqrt{5}, \\
v^{(6)}=\frac{193}{54}, & v^{(7)}=\frac{19783-22428 \cos \frac{\pi}{7}+16380 \cos \frac{2 \pi}{7}-29903 \cos \frac{3 \pi}{7}}{42 \sin ^{2} \frac{2 \pi}{7}} .
\end{array}
$$

Naively, these poles should be canceled by the D2 2-instanton. At this order, however, there might be another contribution that we will discuss in the next section, i.e., the contribution from a kind of bound states of the D2-instanton and the worldsheet instanton. At present, we cannot conclude whether such a contribution exists or not. If exists, we need to consider its pole structure. We leave this problem as a future work.

\section{Discussions}

In this paper, by generalizing our previous method we continued the exact computation of the partition function in the ABJM theory using the Fermi gas formalism. Using these exact values of the partition functions, we further studied the instanton effects. We have found that the grand potential contains an interesting oscillatory behavior and it can be explained by the periodicity of the grand partition function. The worldsheet instanton effects are understood from the results in the topological string on local $\mathbb{F}_{0}$. After subtracting the worldsheet instanton, we studied the D2-instanton effects, only little of which has been known so far. We proposed an analytic expression of the D2 1-instanton correction which is consistent with all of the known properties so far. We would like to discuss some open questions in our analysis.

In our previous work [13], we have found that the partition functions $Z_{1}(N)$ at $k=1$ are written as a polynomial of $\frac{1}{\pi}$ with rational coefficients. Here we have studied the partition functions for a few other $k$ 's. We find that at $k=2$ the partition function are polynomials of $\frac{1}{\pi^{2}}$, at $k=3$, polynomials of $\frac{1}{\pi}$ and $\frac{1}{\sqrt{3}}$, at $k=4$, polynomials of $\frac{1}{\pi}$ and at $k=6$, polynomials of $\frac{1}{\sqrt{3} \pi}$. We note in passing a curious observation about the zeros of these polynomials. For example, if we replace $\frac{1}{\pi}$ by a variable $x$ in the partition function $Z_{1}(N)$ at $k=1$, it becomes a polynomial of $x$ of degree $\left[\frac{N}{2}\right]$. If we order the zeros of this polynomial by their absolute values, the first few zeros are very close to $\frac{1}{\pi},-\frac{3}{\pi}, \frac{5}{\pi}$, and so on. We observed a similar pattern of zeros for other $k$ 's. We would like to understand the general structure of $Z_{k}(N)$ better.

We have determined the analytic expression of the leading D2-instanton correction. We would like to study this effect from the worldvolume theory of the Euclidean D2-brane wrapping on $\mathbb{R P}^{3}$. We expect that the prefactor of D2-instanton correction is coming from 
the 1-loop determinant of the fluctuation around the classical D2-brane action. To analyze such effects, the recent result of reproducing the 1-loop log term from the gravitational computation in [29] might be a helpful guide.

Although the worldsheet instanton coefficients $d_{k}^{(n)}$ obtained from the topological string correctly reproduce the 't Hooft limit of free energy studied in [6], we find some discrepancy between the prediction of $d_{k}^{(n)}$ and our results (1.5) obtained by the numerical analysis. For example, let us take a closer look at the $k=3,6$ cases in (1.5). The coefficients of the first two terms in $J_{3}^{(\mathrm{np})}$ and $J_{6}^{(\mathrm{np})}$ agree with the value of $d_{k}^{(1)}$ and $d_{k}^{(2)}$ in (1.3). However, the fourth term of $J_{3}^{(\mathrm{np})}$ and $J_{6}^{(\mathrm{np})}$ is different from $d_{3}^{(4)}=d_{6}^{(4)}=-8$ predicted from the topological string (1.3). Also, for the $k=4$ case, the third term of $J_{4}^{(\mathrm{np})}$ is different from the prediction $d_{4}^{(3)}=\frac{10}{3}$, while the first term agrees with $d_{4}^{(1)}=1$. We believe that the predictions of worldsheet instanton corrections from the topological string (1.3) are correct at generic $k$, and we conjecture that the reason of this discrepancy is due to a kind of bound states of the D2-instanton and the worldsheet instanton. This conjecture is based on the observation that after a few worldsheet instanton terms which agree with $d_{k}^{(n)}$, the discrepancy arises just after the first D2-instanton term appeared in $J_{k}^{(\mathrm{np})}$. We leave more detailed study of the origin of this discrepancy as a future problem.

Recently, a very interesting relation between the sphere partition functions and the non-perturbative completion of topological string partition functions is proposed in [30]. This proposal is reminiscent of the relation between the canonical and the grand canonical partition functions (3.15). As discussed in [9], it is natural to identify the grand partition function $\Xi(\mu)$ of ABJM theory with the non-perturbative completion of the topological string partition function on local $\mathbb{F}_{0}$. It would be interesting to study this relation further.

\section{Acknowledgments}

We are grateful to Edna Cheung, Yang-Hui He, Shinji Hirano, Hiroaki Kanno, Nakwoo Kim, Gregory Korchemsky, Kimyeong Lee, Tomoki Nosaka, Jaemo Park, Zhao Peng, Ryu Sasaki, Rak-Kyeong Seong, Masaki Shigemori, Fumihiko Sugino, Shigeki Sugimoto, Masahito Yamazaki for useful discussions. The work of Y.H. is supported in part by the JSPS Research Fellowship for Young Scientists, while the work of K.O. is supported in part by JSPS Grant-in-Aid for Young Scientists (B) \#23740178.

\section{A Proof of (2.22) for general Hankel matrices}

In this appendix, we will prove the identity (2.22) assuming that a Hankel matrix has the following integral representation

$$
H_{m, n}=\frac{1}{4 \pi} \int_{-1}^{1} d t w(t) t^{m+n}
$$

We also assume that the weight $w(t)$ is a positive even function of $t$ on the interval $(-1,1)$

$$
w(-t)=w(t)>0, \quad t \in(-1,1) .
$$


By the change of variable

$$
t=t_{q} \equiv \tanh \frac{q}{2},
$$

the matrix element $H_{m, n}$ is rewritten as

$$
H_{m, n}=\int_{-\infty}^{\infty} \frac{d q}{2 \pi} \rho_{m}(q) \rho_{n}(q), \quad \rho_{n}(q)=\frac{\sqrt{w\left(t_{q}\right)}}{\sqrt{2} \cosh \frac{q}{2}} t_{q}^{n},
$$

and the trace becomes

$$
\operatorname{Tr} H^{\ell}=\int_{-\infty}^{\infty} \prod_{i=1}^{\ell} \frac{d q_{i}}{2 \pi} \rho\left(q_{1}, q_{2}\right) \cdots \rho\left(q_{\ell}, q_{1}\right)=\operatorname{Tr} \rho^{\ell},
$$

where

$$
\rho\left(q, q^{\prime}\right)=\sum_{n=0}^{\infty} \rho_{n}(q) \rho_{n}\left(q^{\prime}\right)=\frac{\sqrt{w\left(t_{q}\right) w\left(t_{q^{\prime}}\right)}}{2 \cosh \frac{q-q^{\prime}}{2}} .
$$

Let us prove the identity (2.22) for this class of matrix

$$
\frac{\operatorname{det}\left(1+z H_{-}\right)}{\operatorname{det}\left(1-z H_{+}\right)}=H(z)
$$

where $H(z)$ is defined by

$$
H(z)=\sum_{m=0}^{\infty}\left(1-z H_{+}\right)_{0, m}^{-1}=\left\langle e_{0}\left|\frac{1}{1-z H_{+}}\right| v\right\rangle .
$$

with $\left|e_{0}\right\rangle$ and $|v\rangle$ given in (2.23).

\section{A.1 Proof of the even part}

First we consider the even part of (A.7)

$$
\frac{\operatorname{det}\left(1-z^{2} H_{-}^{2}\right)}{\operatorname{det}\left(1-z^{2} H_{+}^{2}\right)}=H(z) H(-z) .
$$

As discussed in our previous paper [13], using the relation $H_{-}^{2}=H_{+}\left(1-\left|e_{0}\right\rangle\left\langle e_{0}\right|\right) H_{+}$the left hand side of (A.9) is written as

$$
\frac{\operatorname{det}\left(1-z^{2} H_{-}^{2}\right)}{\operatorname{det}\left(1-z^{2} H_{+}^{2}\right)}=\left\langle e_{0}\left|\frac{1}{1-z^{2} H_{+}^{2}}\right| e_{0}\right\rangle .
$$

By applying the lemma of Tracy-Widom to the kernel

$$
\rho_{+}\left(q, q^{\prime}\right)=\frac{\rho\left(q, q^{\prime}\right)+\rho\left(q,-q^{\prime}\right)}{2}=\frac{E(q) E\left(q^{\prime}\right)}{\cosh q+\cosh q^{\prime}}, \quad E(q)=\sqrt{w\left(t_{q}\right)} \cosh \frac{q}{2},
$$

we find

$$
\rho_{+}^{2 \ell+1}\left(q, q^{\prime}\right)=\frac{E(q) E\left(q^{\prime}\right)}{\cosh q+\cosh q^{\prime}} \sum_{k=0}^{2 \ell}(-1)^{k} \phi^{k}(q) \phi^{2 \ell-k}\left(q^{\prime}\right) .
$$


The left hand side of (A.12) is rewritten as

$$
\begin{aligned}
\rho_{+}^{2 \ell+1}\left(q, q^{\prime}\right) & =\int \frac{d q_{1}}{2 \pi} \cdots \int \frac{d q_{2 \ell}}{2 \pi} \rho_{+}\left(q, q_{1}\right) \cdots \rho_{+}\left(q_{2 \ell}, q^{\prime}\right) \\
& =\sum_{m=0}^{\infty} \cdots \sum_{n=0}^{\infty} \int \frac{d q_{1}}{2 \pi} \cdots \int \frac{d q_{2 \ell}}{2 \pi} \rho_{2 m}(q) \rho_{2 m}\left(q_{1}\right) \cdots \rho_{2 n}\left(q_{2 \ell}\right) \rho_{2 n}\left(q^{\prime}\right) \\
& =\sum_{m, n=0}^{\infty} \rho_{2 m}(q)\left(H_{+}^{2 \ell}\right)_{m, n} \rho_{2 n}\left(q^{\prime}\right),
\end{aligned}
$$

and $\phi^{k}(q)$ in (A.12) is defined recursively

$$
\phi^{k}(q)=\frac{1}{E(q)} \int \frac{d q^{\prime}}{2 \pi} \rho_{+}\left(q, q^{\prime}\right) E\left(q^{\prime}\right) \phi^{k-1}\left(q^{\prime}\right) .
$$

If we expand $\phi^{k}(q)$ in terms of $\rho_{2 n}(q)$

$$
\phi^{k}(q)=\frac{\sqrt{2}}{E(q)} \sum_{n=0}^{\infty} \rho_{2 n}(q) \alpha_{n}^{(k)}=\frac{1}{\cosh ^{2} \frac{q}{2}} \sum_{n=0}^{\infty} t_{q}^{2 n} \alpha_{n}^{(k)},
$$

then $\alpha_{n}^{(k)}$ satisfies

$$
\alpha_{n}^{(k)}=\sum_{m=0}^{\infty}\left(H_{+}\right)_{n, m} \alpha_{m}^{(k-1)}
$$

The initial condition $\phi^{0}(q)=1$ corresponds to

$$
\alpha_{m}^{(0)}=1 \quad(\forall m) .
$$

In other words, $\alpha_{m}^{(0)}=v_{m}$. From (A.15) and (A.16), $\phi^{k}(q)$ is found to be

$$
\phi^{k}(q)=\frac{1}{\cosh ^{2} \frac{q}{2}} \sum_{n, m=0}^{\infty} t_{q}^{2 n}\left(H_{+}^{k}\right)_{n, m} .
$$

Setting $q=0$ in (A.18), we find

$$
\phi^{k}(0)=\sum_{m=0}^{\infty}\left(H_{+}^{k}\right)_{0, m}=\left\langle e_{0}\left|H_{+}^{k}\right| v\right\rangle .
$$

This implies that $H(z)$ in (A.8) can also be written as

$$
H(z)=\sum_{n=0}^{\infty} \phi^{n}(0) z^{n} .
$$

Finally, taking the limit $q, q^{\prime} \rightarrow 0$ in (A.12) we find

$$
\left(H_{+}^{2 \ell}\right)_{0,0}=\left\langle e_{0}\left|H_{+}^{2 \ell}\right| e_{0}\right\rangle=\sum_{k=0}^{2 \ell}(-1)^{k} \phi^{k}(0) \phi^{2 \ell-k}(0) .
$$

This is the desired relation (A.9). 


\section{A.2 Proof of the odd part}

Let us prove the odd part of (A.8). The kernel $\rho\left(q, q^{\prime}\right)$ can be written as a matrix element in the "auxiliary" quantum mechanical system

$$
\rho\left(q, q^{\prime}\right)=\left\langle q\left|\sqrt{w\left(t_{q}\right)} c_{p} \sqrt{w\left(t_{q}\right)}\right| q^{\prime}\right\rangle, \quad[q, p]=2 \pi i .
$$

In this subsection, we use the notation

$$
c_{q}=\frac{1}{2 \cosh \frac{q}{2}}, \quad s_{q}=\frac{1}{2 \sinh \frac{q}{2}}, \quad t_{q}=\tanh \frac{q}{2} .
$$

Let us consider the following function

$$
\Xi_{1}(z)=\operatorname{det}\left(1+z R_{q} \Pi c_{q} c_{p}\right), \quad R_{q}=w\left(t_{q}\right) c_{q}^{-1} .
$$

Using the commutation relation found in our previous paper [13],

$$
c_{p} c_{q}=-i s_{q} \Pi s_{p}, \quad \Pi=1-\left|0_{p}\right\rangle\left\langle 0_{q}\right|,
$$

we can see that $\Xi_{1}(z)$ is an even function of $z$, as follows. We first rewrite $\Xi_{1}(z)$ as

$$
\begin{aligned}
\Xi_{1}(z) & =\operatorname{det}\left(1+i z R_{q} \Pi s_{p} \Pi^{\dagger} s_{q}\right) \\
& =\operatorname{det}\left(1+i z R_{q} s_{q} \Pi s_{p} \Pi^{\dagger}\right) \\
& =\operatorname{det}\left(1-z R_{q} c_{p} c_{q} \Pi^{\dagger}\right) .
\end{aligned}
$$

Since $\Xi_{1}(z)$ is a real function for $z \in \mathbb{R}$, we find

$$
\Xi_{1}(z)=\Xi_{1}(z)^{\dagger}=\operatorname{det}\left(1-z\left(R_{q} c_{p} c_{q} \Pi^{\dagger}\right)^{\dagger}\right)=\Xi_{1}(-z) .
$$

On the other hand, $\Xi_{1}(z)$ can also be written as

$$
\begin{aligned}
\Xi_{1}(z) & =\operatorname{det}\left(1+z w\left(t_{q}\right) c_{p}\right) \cdot\left\langle 0_{q}\left|R_{q}^{-1} \frac{1}{1+z w\left(t_{q}\right) c_{p}} R_{q}\right| 0_{p}\right\rangle \\
& =\operatorname{det}(1+z \rho) \cdot\left\langle 0_{q}\left|E_{q}^{-1} \frac{1}{1+z \rho} E_{q}\right| 0_{p}\right\rangle,
\end{aligned}
$$

where

$$
E_{q}=\sqrt{w\left(t_{q}\right)} \cosh \frac{q}{2}, \quad \rho=\sqrt{w\left(t_{q}\right)} c_{p} \sqrt{w\left(t_{q}\right)} .
$$

One can easily see that the second factor in the last line of (A.28) is

$$
\left\langle 0_{q}\left|E_{q}^{-1} \frac{1}{1+z \rho} E_{q}\right| 0_{p}\right\rangle=\sum_{n=0}^{\infty} \phi^{n}(0)(-z)^{n}=H(-z) .
$$

Then $\Xi_{1}(-z)=\Xi_{1}(z)$ implies the following relation

$$
\frac{H(z)}{H(-z)}=\frac{\operatorname{det}\left(1+z H_{+}\right) \operatorname{det}\left(1+z H_{-}\right)}{\operatorname{det}\left(1-z H_{+}\right) \operatorname{det}\left(1-z H_{-}\right)} .
$$

This completes the proof of the odd part of (A.7). 


\section{B Exact values of $Z_{k}(N)$}

Here let us summarize the exact values of $Z_{k}(N)(k=1,2,3,4,6)$. Our main task is to solve the integral equation (2.15). Here we use the method in [14]. This method allows us to rewrite the convolution in (2.15) as the sum of the residues.

\section{B.1 Recursive relations at even $k$}

Let us first consider the even $k$ case. Introducing the variables $u=e^{q / k}$ and $v=e^{q^{\prime} / k}$, then the integral equation (2.15) becomes

$$
\varphi^{\ell}(u)=\frac{1}{2 \pi} \frac{u}{u+1} \int_{0}^{\infty} d v \frac{v^{(k-2) / 2}(v+1)}{(u+v)\left(v^{k}+1\right)} \varphi^{\ell-1}(v),
$$

where $\varphi^{\ell}(u)=\phi^{\ell}(q)$. Note that $v^{(k-2) / 2}$ is rational when $k$ is even. From the solutions for lower $\ell$, we observe that the solution $\varphi^{\ell}(u)$ have the following form

$$
\varphi^{\ell}(u)=\sum_{j=0}^{\ell} A_{\ell}^{(j)}(u) \log ^{j} u,
$$

where $A_{\ell}^{(j)}(u)$ is a rational function. We assume that $A_{\ell}^{(j)}(u)$ has the poles at the roots of $(u+1)\left(u^{k}+1\right)=0$. We can rewrite the equation (B.1) by using the technique in [14] as follows,

$$
\begin{aligned}
\varphi^{\ell+1}(u) & =\frac{1}{2 \pi} \frac{u}{u+1} \int_{0}^{\infty} d v \frac{v^{k / 2-1}(v+1)}{(u+v)\left(v^{k}+1\right)} \sum_{j=0}^{\ell} A_{\ell}^{(j)}(v) \log ^{j} v \\
& =-\frac{1}{2 \pi} \frac{u}{u+1} \sum_{j=0}^{\ell} \frac{(2 \pi i)^{j}}{j+1} \oint_{\gamma} d v \frac{v^{k / 2-1}(v+1)}{(u+v)\left(v^{k}+1\right)} A_{\ell}^{(j)}(v) B_{j+1}\left(\frac{\log v}{2 \pi i}\right) \\
& =-\frac{1}{2 \pi} \frac{u}{u+1} \sum_{j=0}^{\ell} \frac{(2 \pi i)^{j+1}}{j+1} \sum_{\text {all poles }} \operatorname{Res}_{v} \frac{v^{(k-2) / 2}(v+1)}{(u+v)\left(v^{k}+1\right)} A_{\ell}^{(j)}(v) B_{j+1}\left(\frac{\log v}{2 \pi i}\right) \\
& =-\frac{(-u)^{k / 2}(u-1)}{2 \pi(u+1)\left(u^{k}+1\right)} \sum_{j=0}^{\ell} \frac{(2 \pi i)^{j+1}}{j+1} A_{\ell}^{(j)}(-u) B_{j+1}\left(\frac{\log u}{2 \pi i}+\frac{1}{2}\right)+f_{\ell}(u),
\end{aligned}
$$

where $B_{n}(x)$ is the Bernoulli polynomial, and $f_{\ell}(u)$ is a rational function. In the second line in (B.3), we have used the integral formula noted in [14],

$$
\int_{0}^{\infty} d v C(v) \log ^{j} v=-\frac{(2 \pi i)^{j}}{j+1} \oint_{\gamma} d v C(v) B_{j+1}\left(\frac{\log v}{2 \pi i}\right),
$$

where we choose the branch cut of log as the positive real axis, and integration contour $\gamma$ is chosen as the closed path from $+\infty$ to 0 infinitesimally below the cut and then from 0 to $+\infty$ infinitesimally above the cut. Using the identity:

$$
B_{n}(x+a)=\sum_{p=0}^{n}\left(\begin{array}{l}
n \\
p
\end{array}\right) B_{n-p}(a) x^{p},
$$


the first term of the right hand side in (B.3) becomes

$$
\begin{aligned}
& -\frac{(-u)^{k / 2}(u-1)}{2 \pi(u+1)\left(u^{k}+1\right)} \sum_{j=0}^{\ell} \sum_{p=0}^{j+1} \frac{(2 \pi i)^{j+1}}{j+1}\left(\begin{array}{c}
j+1 \\
p
\end{array}\right) B_{j+1-p}\left(\frac{1}{2}\right) A_{\ell}^{(j)}(-u)\left(\frac{\log u}{2 \pi i}\right)^{p} \\
& =-\frac{(-u)^{k / 2}(u-1)}{2 \pi(u+1)\left(u^{k}+1\right)} \sum_{p=0}^{\ell} \sum_{j=0}^{p+1} \frac{(2 \pi i)^{p+1}}{p+1}\left(\begin{array}{c}
p+1 \\
j
\end{array}\right) B_{p+1-j}\left(\frac{1}{2}\right) A_{\ell}^{(p)}(-u)\left(\frac{\log u}{2 \pi i}\right)^{j} \\
& =-\frac{(-u)^{k / 2}(u-1)}{2 \pi(u+1)\left(u^{k}+1\right)} \sum_{j=0}^{\ell+1} \sum_{\substack{p=j-1 \\
p \geq 0}}^{\ell} \frac{(2 \pi i)^{p+1-j}}{p+1}\left(\begin{array}{c}
p+1 \\
j
\end{array}\right) B_{p+1-j}\left(\frac{1}{2}\right) A_{\ell}^{(p)}(-u) \log ^{j} u
\end{aligned}
$$

We note that $B_{p+1-j}(1 / 2)$ vanishes if $p+1-j$ is odd. Thus setting $p+1-j=2 m$, we obtain

$$
-\frac{(-u)^{k / 2}(u-1)}{(u+1)\left(u^{k}+1\right)} \sum_{j=0}^{\ell+1} \sum_{\substack{m=0 \\
j+2 m-1 \geq 0}}^{\left[\frac{\ell-j+1}{2}\right]} \frac{(-1)^{m}(2 \pi)^{2 m-1}}{j+2 m}\left(\begin{array}{c}
j+2 m \\
j
\end{array}\right) B_{2 m}\left(\frac{1}{2}\right) A_{\ell}^{(j+2 m-1)}(-u) \log ^{j} u .
$$

Comparing the coefficients of $\log ^{j} u$, we obtain the recursive relations for $A_{\ell}^{(j)}(u)$.

For $1 \leq j \leq \ell+1$, we find

$$
A_{\ell+1}^{(j)}(u)=-\frac{(-u)^{k / 2}(u-1)}{(u+1)\left(u^{k}+1\right)} \sum_{m=0}^{\left[\frac{\ell-j+1}{2}\right]} \frac{(-1)^{m}(2 \pi)^{2 m-1}}{j+2 m}\left(\begin{array}{c}
j+2 m \\
j
\end{array}\right) B_{2 m}\left(\frac{1}{2}\right) A_{\ell}^{(j+2 m-1)}(-u),
$$

while for $j=0$ we find

$$
A_{\ell+1}^{(0)}(u)=-\frac{(-u)^{k / 2}(u-1)}{(u+1)\left(u^{k}+1\right)} \sum_{m=1}^{\left[\frac{\ell+1}{2}\right]} \frac{(-1)^{m}(2 \pi)^{2 m-1}}{2 m} B_{2 m}\left(\frac{1}{2}\right) A_{\ell}^{(2 m-1)}(-u)+f_{\ell}(u),
$$

where the rational function $f_{\ell}(u)$ is given by

$$
f_{\ell}(u)=-\frac{u}{2 \pi(u+1)} \sum_{j=0}^{\ell} \frac{(2 \pi i)^{j+1}}{j+1} \sum_{v \neq-u} \operatorname{Res} \frac{v^{k / 2-1}(v+1)}{(u+v)\left(v^{k}+1\right)} A_{\ell}^{(j)}(v) B_{j+1}\left(\frac{\log v}{2 \pi i}\right) .
$$

Here $\sum_{v \neq-u}$ means the sum over all the poles except for $v=-u$. It is convenient to redefine

$$
A_{\ell}^{(j)}(u)=\frac{1}{(2 \pi)^{j} j !}\left(\frac{u^{k / 2}}{u^{k}+1}\right)^{j} \hat{A}_{\ell}^{(j)}(u)
$$


Then we can rewrite the recursive relations as

$$
\begin{aligned}
& \hat{A}_{\ell+1}^{(j)}(u)=(-1)^{k j / 2+1} \frac{u-1}{u+1} \sum_{m=0}^{\left[\frac{\ell-j+1}{2}\right]} c_{m}\left(\frac{u^{k / 2}}{u^{k}+1}\right)^{2 m} \hat{A}_{\ell}^{(j+2 m-1)}(-u), \quad(1 \leq j \leq \ell+1), \\
& \hat{A}_{\ell+1}^{(0)}(u)=-\frac{u-1}{u+1} \sum_{m=1}^{\left[\frac{\ell+1}{2}\right]} c_{m}\left(\frac{u^{k / 2}}{u^{k}+1}\right)^{2 m} \hat{A}_{\ell}^{(2 m-1)}(-u)+f_{\ell}(u),
\end{aligned}
$$

where

$$
\begin{aligned}
c_{m} & =\frac{(-1)^{m}}{(2 m) !} B_{2 m}\left(\frac{1}{2}\right)=\frac{(-1)^{m}}{(2 m) !}\left(\frac{1}{2^{2 m-1}}-1\right) B_{2 m} \\
f_{\ell}(u) & =-\frac{u}{u+1} \sum_{j=0}^{\ell} \frac{i^{j+1}}{(j+1) !} \sum_{v \neq-u} \operatorname{Res} \frac{v+1}{v(u+v)}\left(\frac{v^{k / 2}}{v^{k}+1}\right)^{j+1} \hat{A}_{\ell}^{(j)}(v) B_{j+1}\left(\frac{\log v}{2 \pi i}\right) .
\end{aligned}
$$

The initial condition is trivially given by

$$
\hat{A}_{0}^{(0)}(u)=1 .
$$

The equations (B.11)-(B.15) are the recursive relations to be solved.

Once these equations are solved, we obtain $\varphi^{\ell}(u)$, and thus can compute $\phi^{\ell}(0)=\varphi^{\ell}(1)$ and $\operatorname{Tr} \rho_{+}^{2 n}$. The matrix element $\rho_{+}^{2 n}\left(u_{1}, u_{2}\right)$ is given by

$$
\rho_{+}^{2 n}\left(u_{1}, u_{2}\right)=F_{k}\left(u_{1}, u_{2}\right) \sum_{\ell=0}^{2 n-1}(-1)^{\ell} \varphi^{\ell}\left(u_{1}\right) \varphi^{2 n-1-\ell}\left(u_{2}\right),
$$

where

$$
F_{k}\left(u_{1}, u_{2}\right)=\frac{\left(u_{1} u_{2}\right)^{\frac{k}{4}+\frac{1}{2}}\left(u_{1}+1\right)\left(u_{2}+1\right)}{2 k\left(u_{1}-u_{2}\right)\left(u_{1} u_{2}-1\right)\left(u_{1}^{k}+1\right)^{1 / 2}\left(u_{2}^{k}+1\right)^{1 / 2}} .
$$

The trace of $\rho_{+}^{2 n}$ is then computed by

$$
\operatorname{Tr} \rho_{+}^{2 n}=\int \frac{d q}{2 \pi} \rho_{+}^{2 n}(q, q)=\frac{k}{2 \pi} \int_{0}^{\infty} d u \frac{\rho_{+}^{2 n}(u, u)}{u} .
$$

It is easy to see that $\rho_{+}^{2 n}(u, u) / u$ has the following form

$$
\frac{\rho_{+}^{2 n}(u, u)}{u}=\sum_{j=0}^{2 n-1} R_{2 n}^{(j)}(u) \log ^{j} u .
$$

where $R_{2 n}^{(j)}(u)$ is a rational function. From the computation for low $n$ 's, we observe that $R_{2 n}^{(j)}(u)$ has the poles at the roots of $u^{2 k}-1=0$. We assume that this observation is valid for arbitrary $n$. Let $u_{m}$ be the root of $u^{2 k}-1=0$,

$$
u_{m}=\cos \left(\frac{\pi m}{k}\right)+i \sin \left(\frac{\pi m}{k}\right), \quad(m=0, \pm 1, \pm 2, \ldots) .
$$


Then, $\operatorname{Tr} \rho_{+}^{2 n}$ is computed as

$$
\begin{aligned}
\operatorname{Tr} \rho_{+}^{2 n} & =\frac{k}{2 \pi} \int_{0}^{\infty} d u \sum_{j=0}^{2 n-1} R_{2 n}^{(j)}(u) \log ^{j} u \\
& =-\frac{k}{2 \pi} \sum_{j=0}^{2 n-1} \frac{(2 \pi i)^{j+1}}{j+1} \sum_{m=0}^{2 k-1} \operatorname{Res}_{u=u_{m}} R_{2 n}^{(j)}(u) B_{j+1}\left(\frac{\log u}{2 \pi i}\right) .
\end{aligned}
$$

\section{B.2 Recursive relations at odd $k$}

Next let us consider the odd $k$ case. Changing the variables to $t=e^{q / 2 k}$ and $s=e^{q^{\prime} / 2 k}$, we obtain

$$
\psi^{\ell}(t)=\frac{1}{\pi} \frac{t^{2}}{t^{2}+1} \int_{0}^{\infty} d s \frac{s^{k-1}\left(s^{2}+1\right)}{\left(t^{2}+s^{2}\right)\left(s^{2 k}+1\right)} \psi^{\ell-1}(s),
$$

where $\psi^{\ell}(t)=\phi^{\ell}(q)$. We observe that the solution takes the form

$$
\psi^{\ell}(t)=\sum_{j=0}^{\left[\frac{\ell}{2}\right]} A_{\ell}^{(j)}(t) \log ^{j} t
$$

where a rational function $A_{\ell}^{(j)}(t)$ has poles at the roots of $t^{4 k}-1=0$. Let $\nu_{m}$ be the roots of $t^{4 k}-1=0$,

$$
\nu_{m}=\cos \left(\frac{m \pi}{2 k}\right)+i \sin \left(\frac{m \pi}{2 k}\right), \quad(m=0 \pm 1, \pm 2, \ldots) .
$$

In the same computation in the previous subsection, we find the recursive relations for $A_{\ell}^{(j)}(t)$,

$$
\begin{aligned}
A_{\ell+1}^{(j)}(t)= & (-1)^{\frac{k+1}{2}} \frac{t^{k}\left(t^{2}-1\right)}{\left(t^{2}+1\right)\left(t^{2 k}-1\right)} \sum_{p=j-1}^{\left[\frac{\ell}{2}\right]} \frac{(2 \pi i)^{p-j}}{p+1}\left(\begin{array}{c}
p+1 \\
j
\end{array}\right) B_{p+1-j}\left(\frac{1}{4}\right) \\
& \times\left[A_{\ell}^{(p)}(i t)+(-1)^{p-j} A_{\ell}^{(p)}(-i t)\right], \quad(1 \leq j \leq[\ell / 2]+1), \\
A_{\ell+1}^{(0)}(t)= & (-1)^{\frac{k+1}{2}} \frac{t^{k}\left(t^{2}-1\right)}{\left(t^{2}+1\right)\left(t^{2 k}-1\right)} \sum_{p=0}^{\left[\frac{\ell}{2}\right]} \frac{(2 \pi i)^{p}}{p+1} B_{p+1}\left(\frac{1}{4}\right)\left[A_{\ell}^{(p)}(i t)+(-1)^{p} A_{\ell}^{(p)}(-i t)\right]+f_{\ell}(t),
\end{aligned}
$$

where

$$
f_{\ell}(t)=-\frac{1}{\pi} \frac{t^{2}}{t^{2}+1} \sum_{j=0}^{\left[\frac{\ell}{2}\right]} \frac{(2 \pi i)^{j+1}}{j+1} \sum_{m=0}^{4 k-1} \operatorname{Res}_{s=\nu_{m}} \frac{s^{k-1}\left(s^{2}+1\right)}{\left(t^{2}+s^{2}\right)\left(s^{2 k}+1\right)} A_{\ell}^{(j)}(s) B_{j+1}\left(\frac{\log s}{2 \pi i}\right) .
$$

The matrix element $\rho_{+}^{2 n}\left(t_{1}, t_{2}\right)$ is given by

$$
\rho_{+}^{2 n}\left(t_{1}, t_{2}\right)=\tilde{F}_{k}\left(t_{1}, t_{2}\right) \sum_{\ell=0}^{2 n-1}(-1)^{\ell} \psi^{\ell}\left(t_{1}\right) \psi^{2 n-1-\ell}\left(t_{2}\right)
$$


where

$$
\tilde{F}_{k}\left(t_{1}, t_{2}\right)=\frac{\left(t_{1} t_{2}\right)^{k / 2+1}\left(t_{1}^{2}+1\right)\left(t_{2}^{2}+1\right)}{2 k\left(t_{1}^{2}-t_{2}^{2}\right)\left(t_{1}^{2} t_{2}^{2}-1\right)\left(t_{1}^{2 k}+1\right)^{1 / 2}\left(t_{2}^{2 k}+1\right)^{1 / 2}} .
$$

We further assume that $\rho_{+}^{2 n}\left(t_{1}, t_{2}\right) / t$ takes the form

$$
\frac{\rho_{+}^{2 n}(t, t)}{t}=\sum_{j=0}^{n-1} R_{2 n}^{(j)}(t) \log ^{j} t,
$$

where a rational function $R_{2 n}^{(j)}(t)$ has poles at the roots of $t^{4 k}-1=0$. Thus $\operatorname{Tr} \rho_{+}^{2 n}$ is computed by

$$
\begin{aligned}
\operatorname{Tr} \rho_{+}^{2 n} & =\frac{k}{\pi} \int_{0}^{\infty} d t \sum_{j=0}^{n-1} R_{2 n}^{(j)}(t) \log ^{j} t \\
& =-\frac{k}{\pi} \sum_{j=0}^{n-1} \frac{(2 \pi i)^{j+1}}{j+1} \sum_{m=0}^{4 k-1} \operatorname{Res}_{t=\nu_{m}} R_{2 n}^{(j)}(t) B_{j+1}\left(\frac{\log t}{2 \pi i}\right) .
\end{aligned}
$$

Let us summarize our procedure here. For even $k$, we solve the recursive relations (B.11)-(B.15), then compute $\operatorname{Tr} \rho_{+}^{2 n}$ by (B.21). For odd $k$, the recursive relations to be solved are (B.25)-(B.26). We can compute $\operatorname{Tr} \rho_{+}^{2 n}$ by (B.30). These equations do not contain any integrals, and we can simply solve by using Mathematica. We have actually solved these equations, and have computed $Z_{k}(N)$ up to $N=44,20,18,16,14$ at $k=1,2,3,4,6$ respectively. These values are summarized in figures $9-13$.

\section{B.3 A guess}

The exact values of the partition functions obtained here are all polynomials of $1 / \pi$. At $k=1$, in particular, the partition function has the following form

$$
Z_{1}(N)=\sum_{\ell=0}^{\left[\frac{N}{2}\right]} \frac{Z_{1}^{(\ell)}(N)}{\pi^{\ell}},
$$

where the coefficients $Z_{1}^{(\ell)}(N)$ are rational numbers. It is, of course, difficult to conjecture all the coefficients $Z_{1}^{(\ell)}(N)$ for general $N$. However, we find that the leading and the next-to-leading coefficients are simply given by

$$
\begin{aligned}
Z_{1}^{(M)}(2 M) & =\frac{1}{M !}\left(\frac{1}{8 \sqrt{2} i}\right)^{M} H_{M}\left(\frac{i}{2 \sqrt{2}}\right), \quad Z_{1}^{(M-1)}(2 M)=0, \\
Z_{1}^{(M)}(2 M+1) & =\frac{1}{4 M !}\left(-\frac{1}{8 \sqrt{2} i}\right)^{M} H_{M}\left(\frac{3 i}{2 \sqrt{2}}\right), \\
Z_{1}^{(M-1)}(2 M+1) & =\frac{1}{64(M-1) !}\left(\frac{1}{8 \sqrt{2} i}\right)^{M-1} H_{M-1}\left(\frac{5 i}{2 \sqrt{2}}\right),
\end{aligned}
$$

where $H_{n}(x)$ is the $n$-th Hermite polynomial. One can check that this guess is correct at least up to $N=44$ by using the values showed in figures 9 and 10 . 


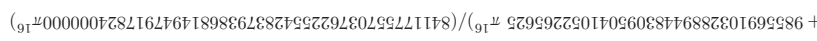

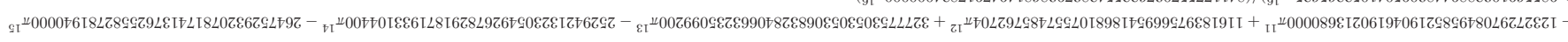

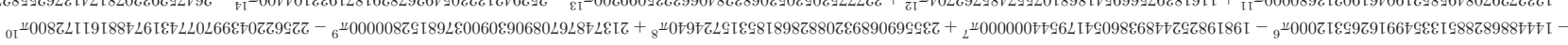

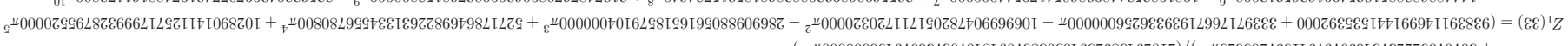

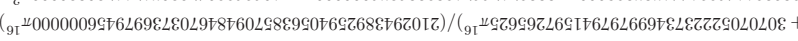

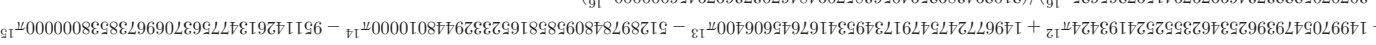

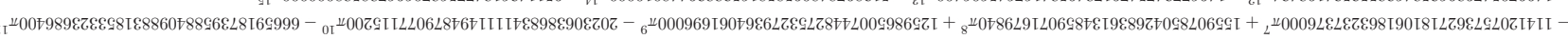

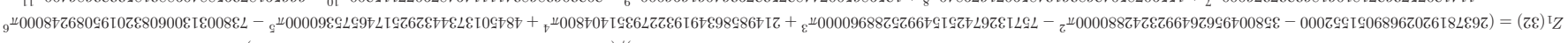

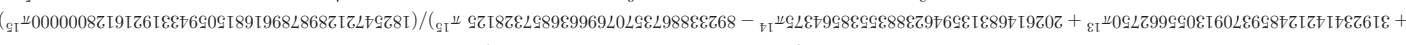

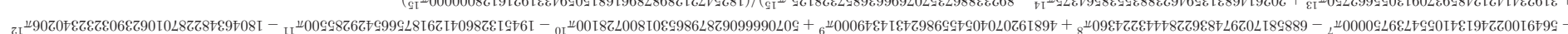

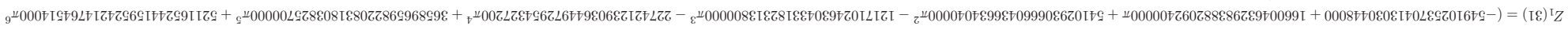

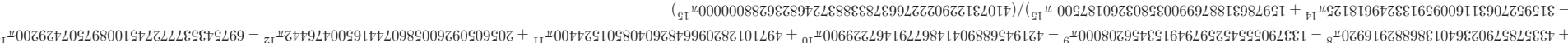

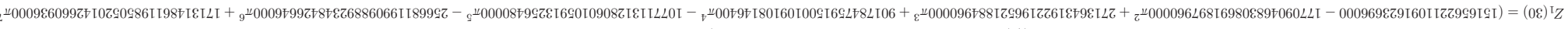

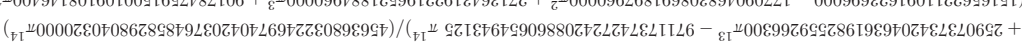

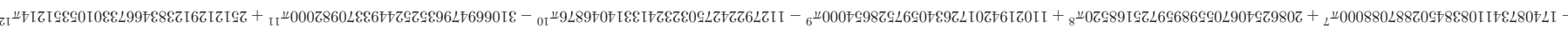

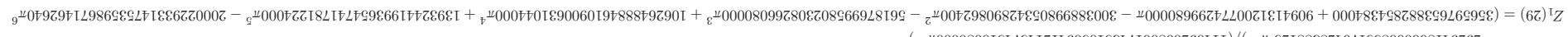

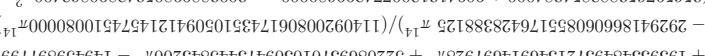

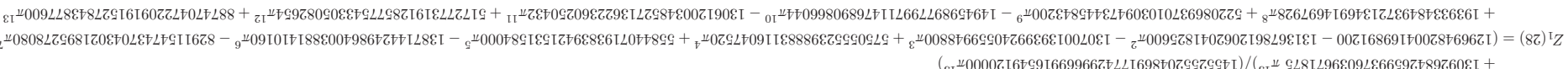

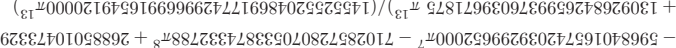

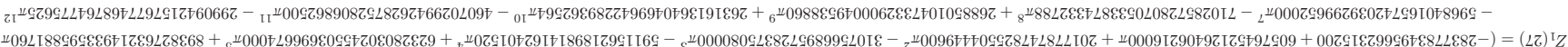

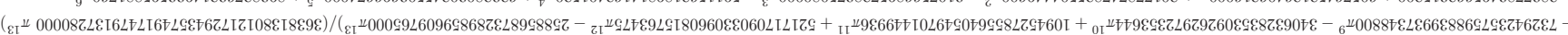

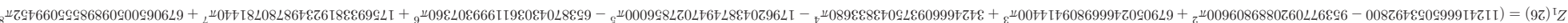

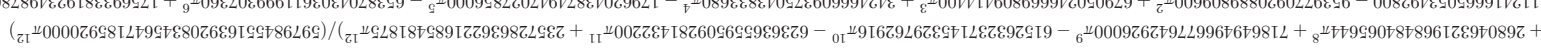

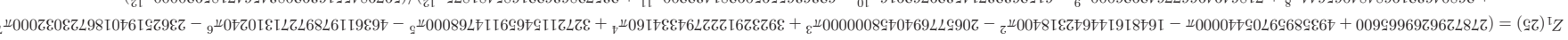

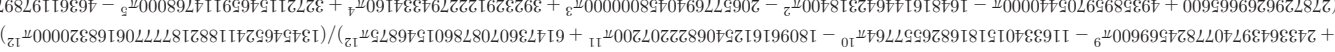

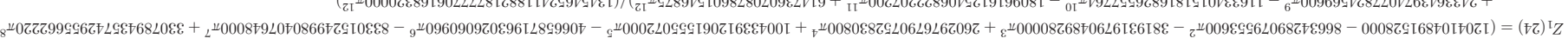

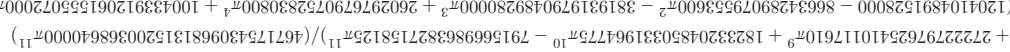

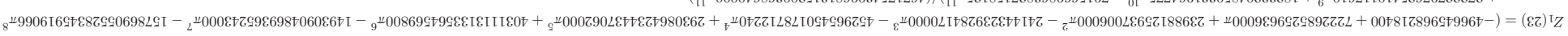

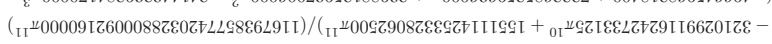

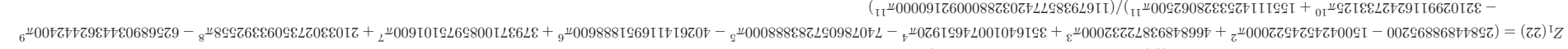

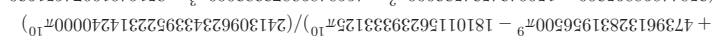

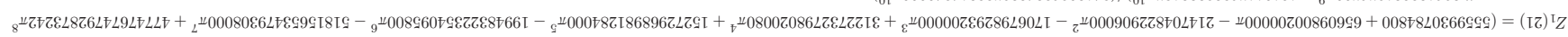

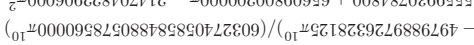

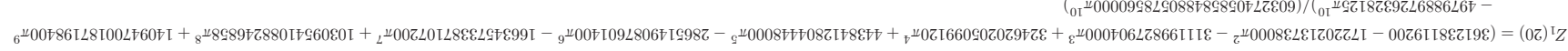

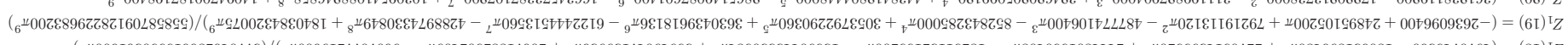

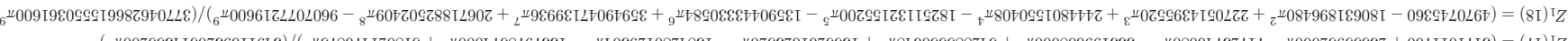
(2) (2)

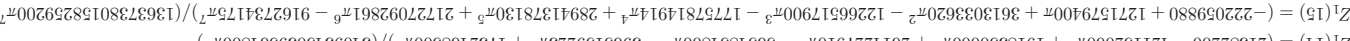
(20

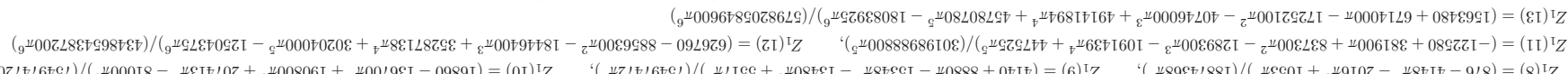

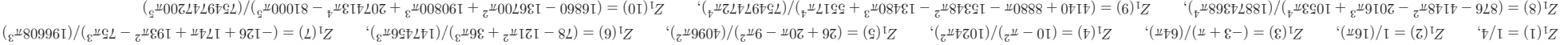




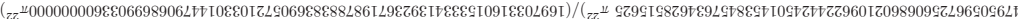

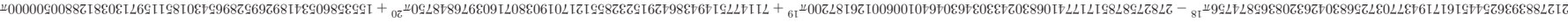
L 006 c92

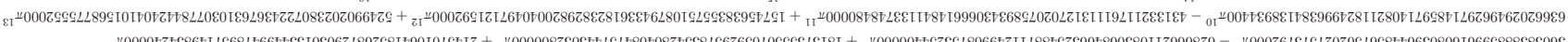

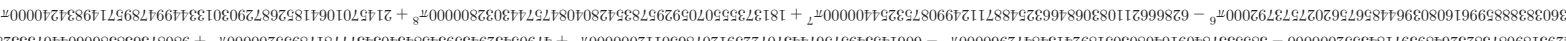

(15

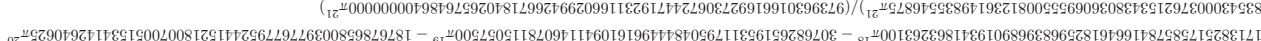

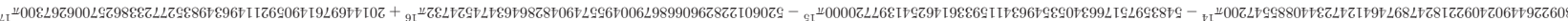

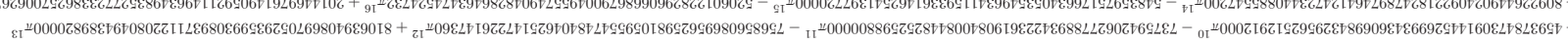

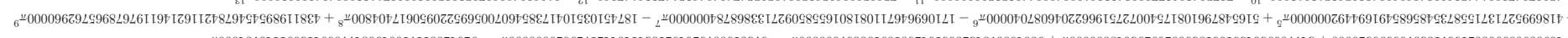
¿

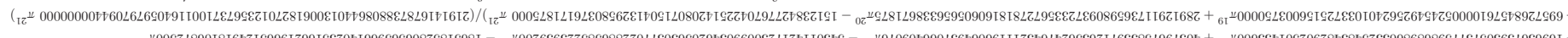

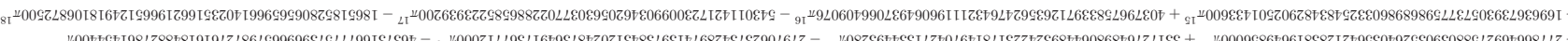

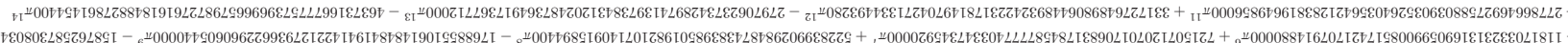

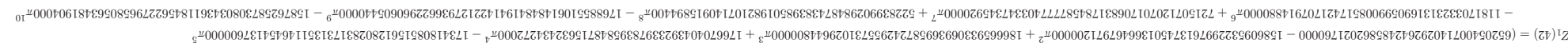
年

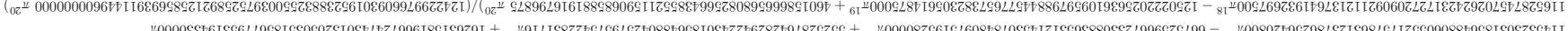
年 政

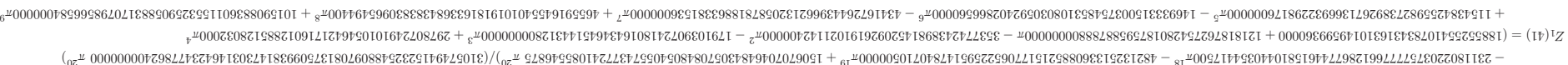

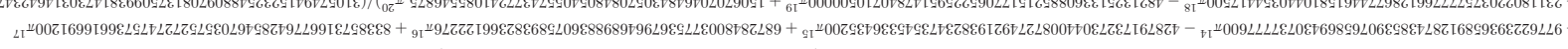

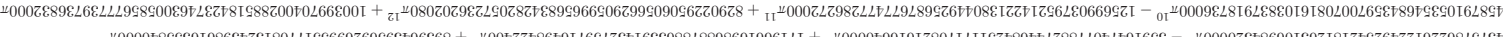
ע.

(6.

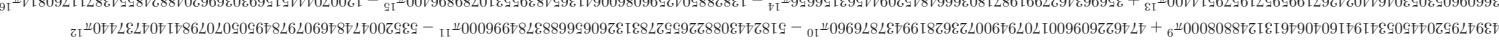

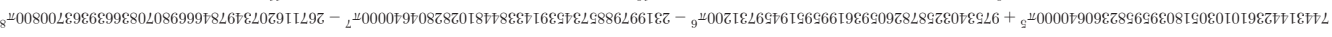

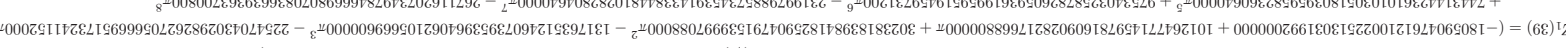

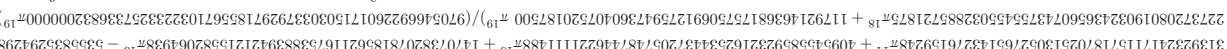
4t

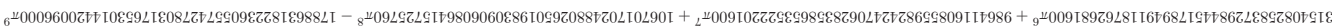
4 G.

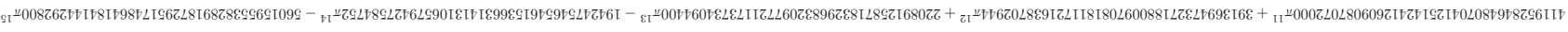

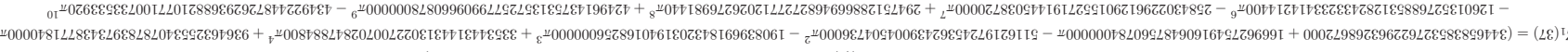
8I

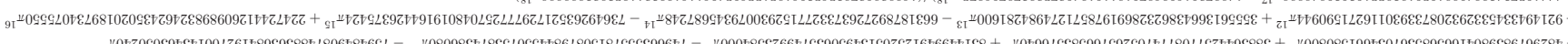

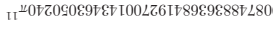
年

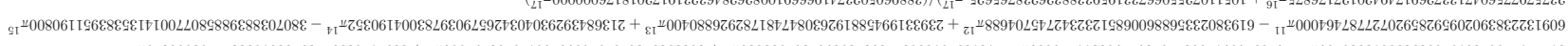

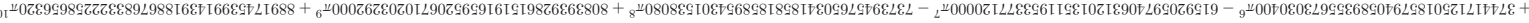
«

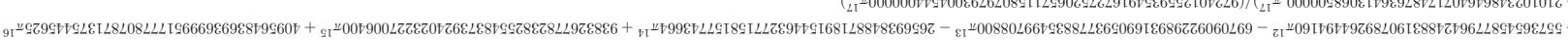
4

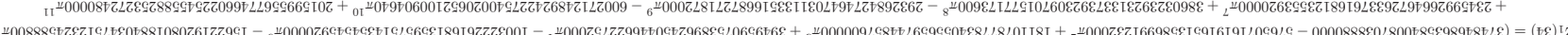




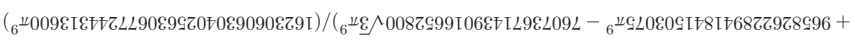

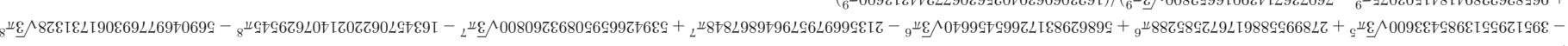

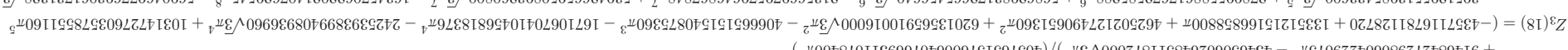

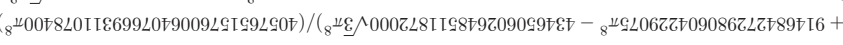

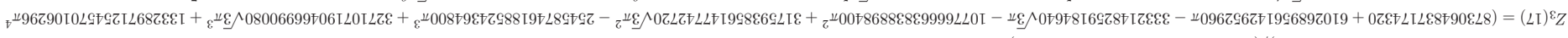

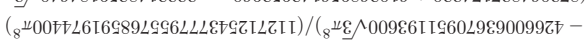

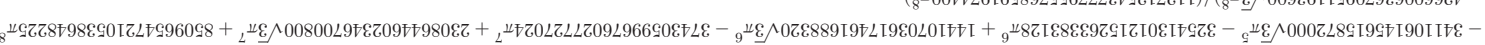

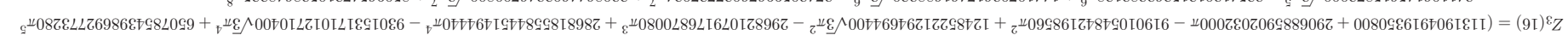

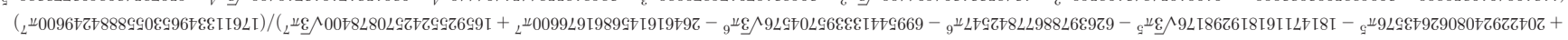

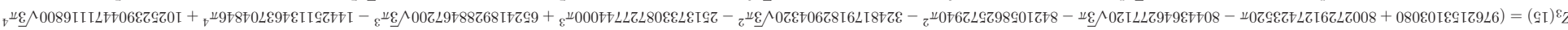

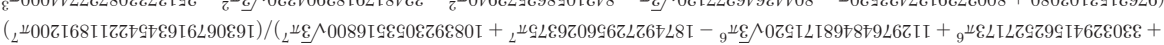

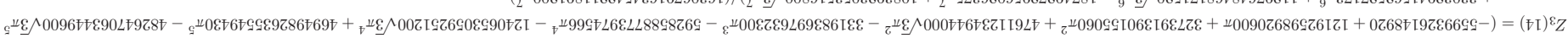

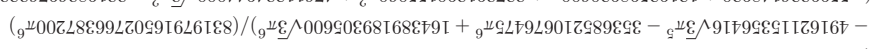

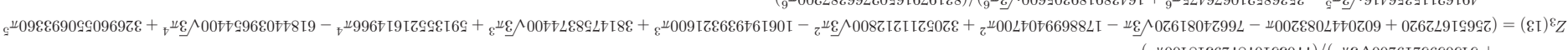

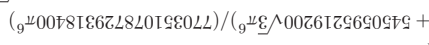

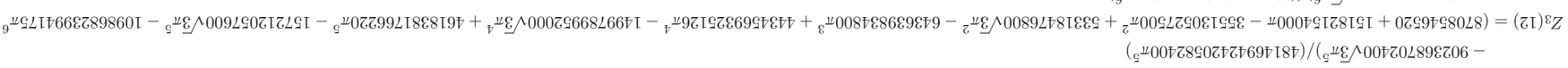

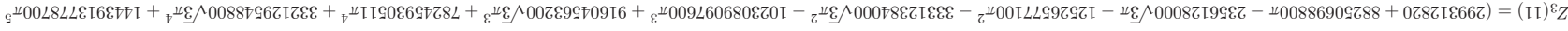

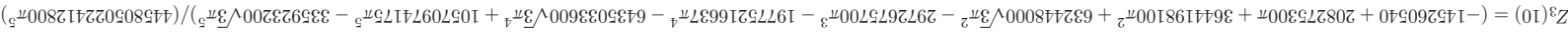

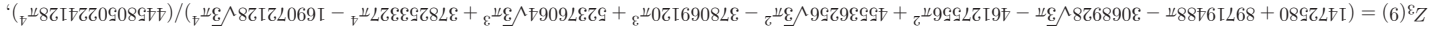

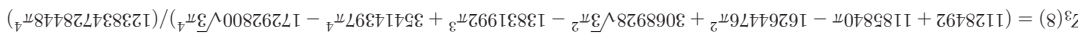

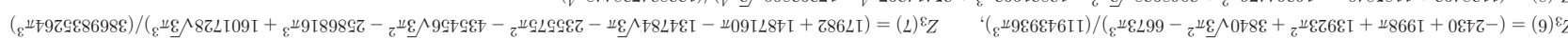

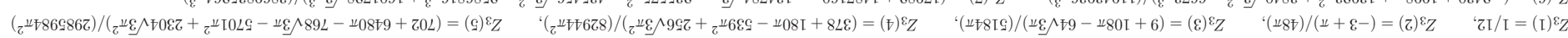

(ов

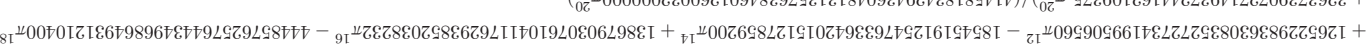

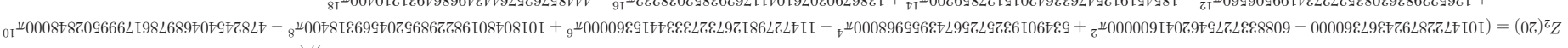

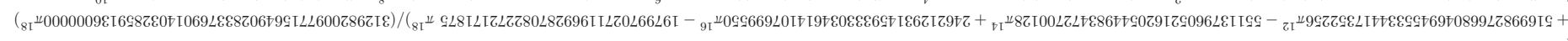

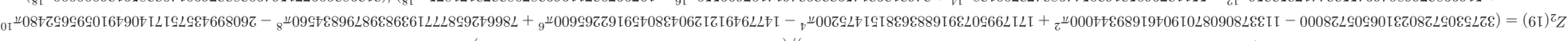

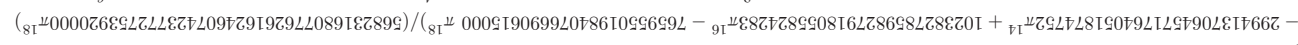

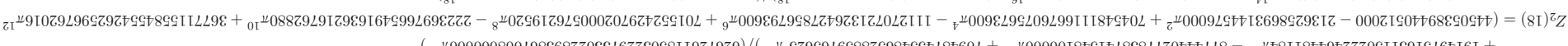

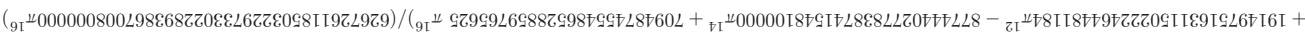

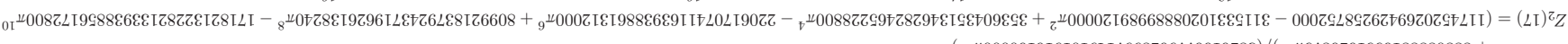

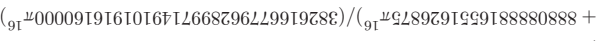

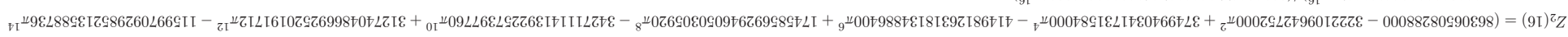

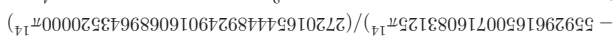

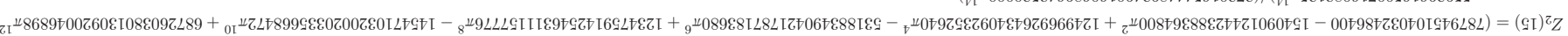

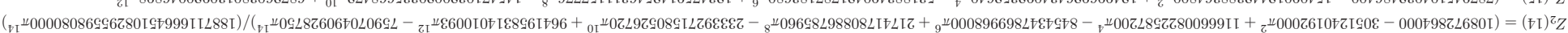

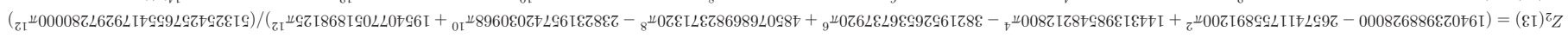

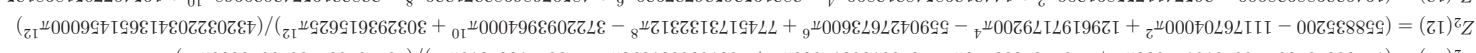

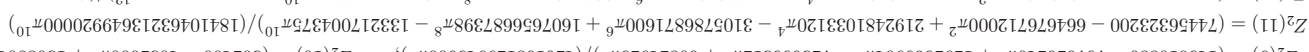

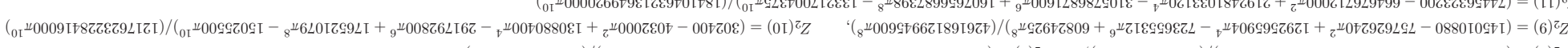

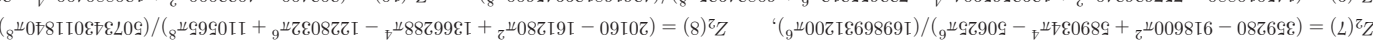

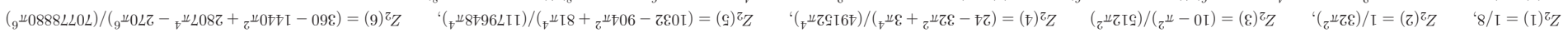




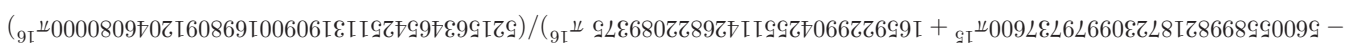

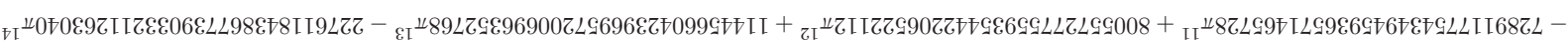

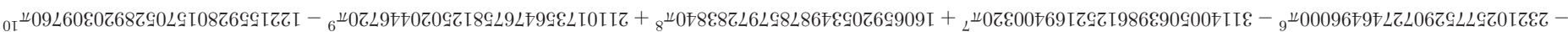

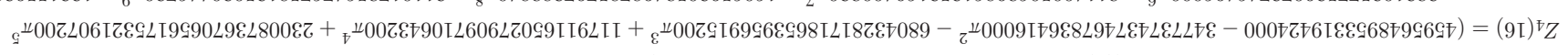

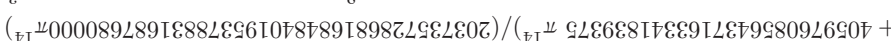

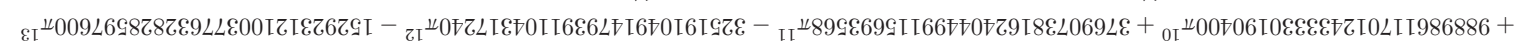

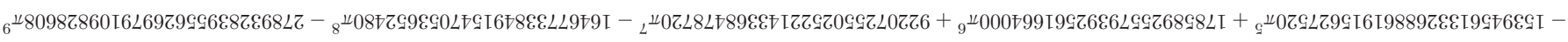

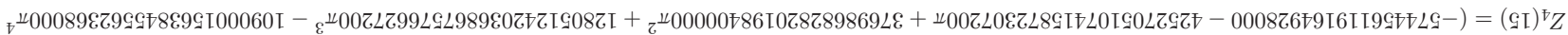

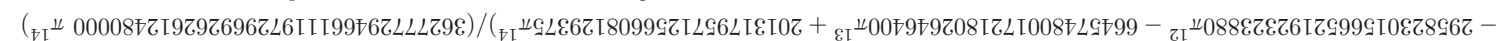

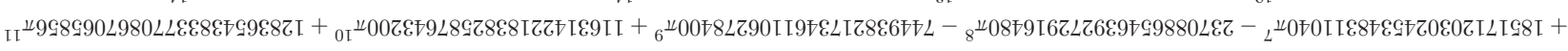

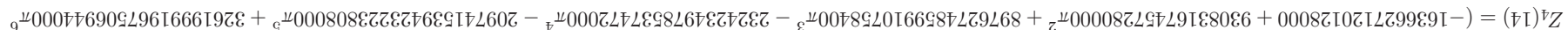

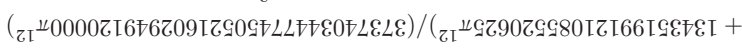

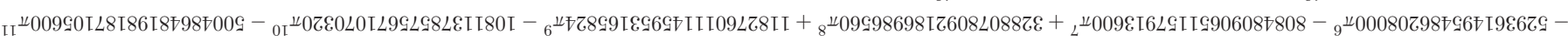

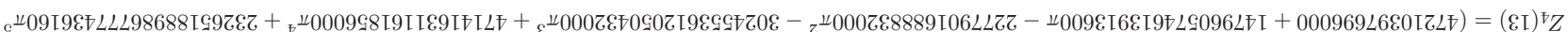

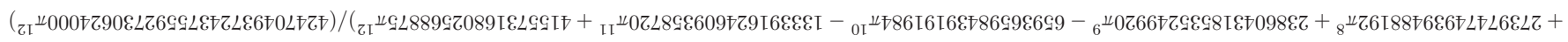

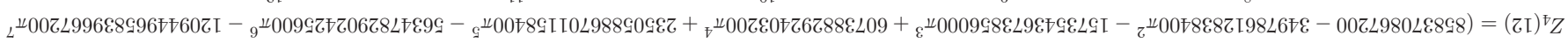

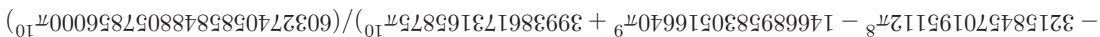

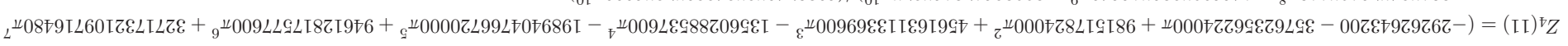

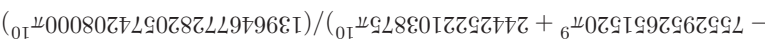

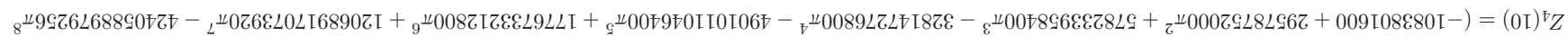

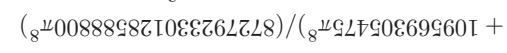

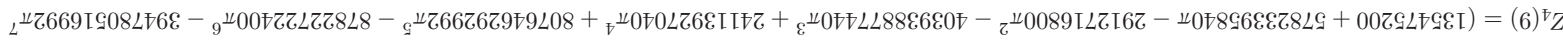

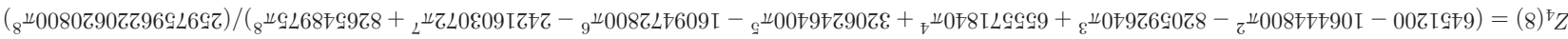

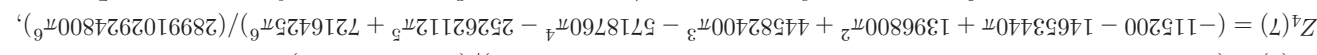

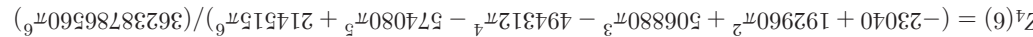

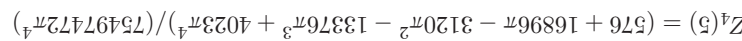

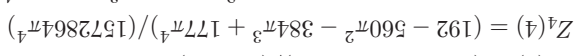

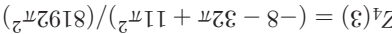

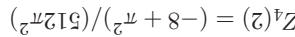




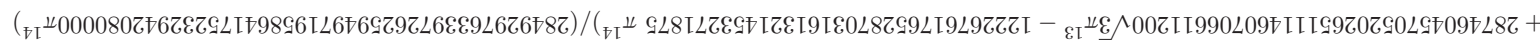

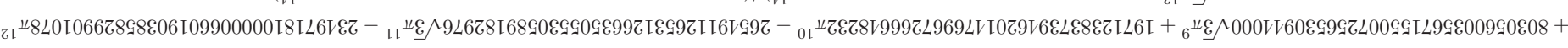

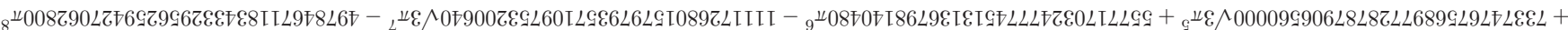

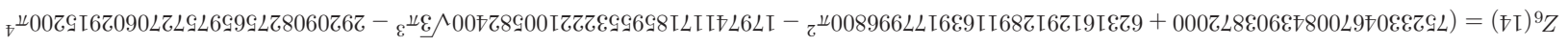

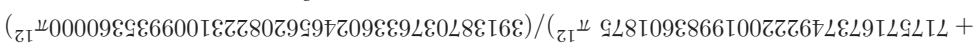

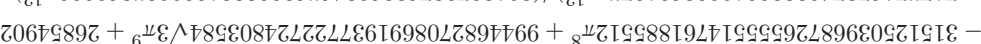

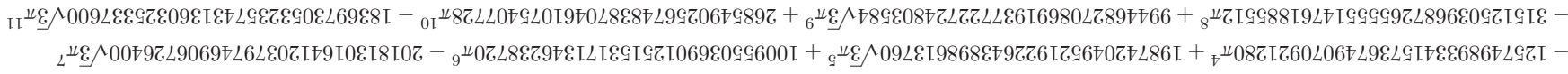

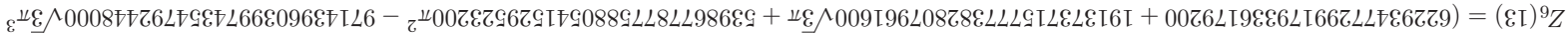

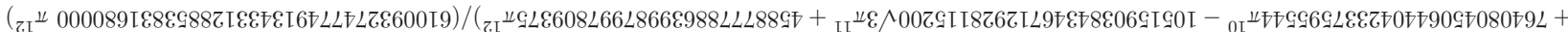

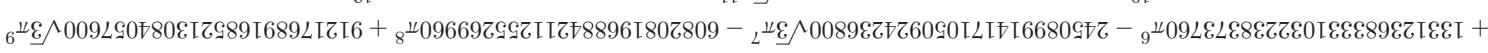

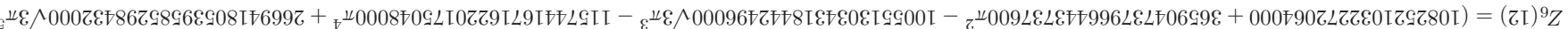

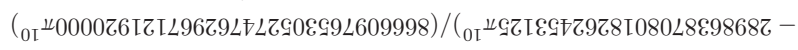

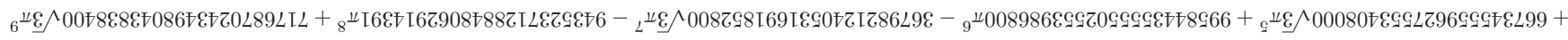

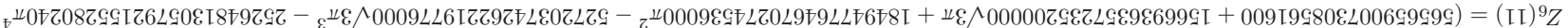

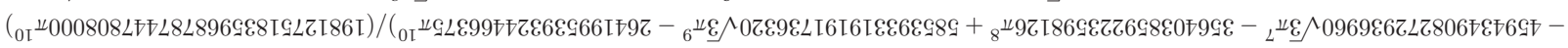

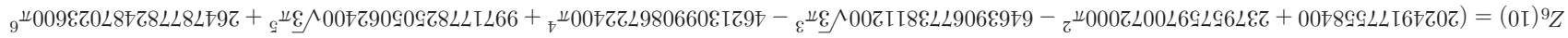

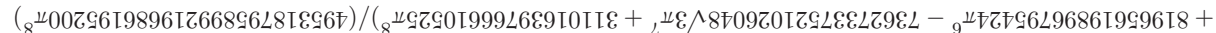

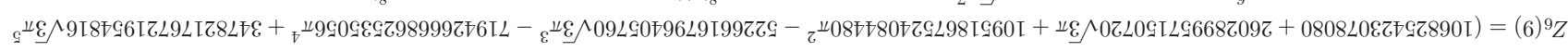

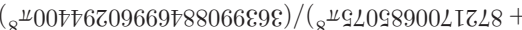

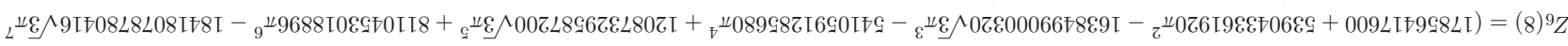

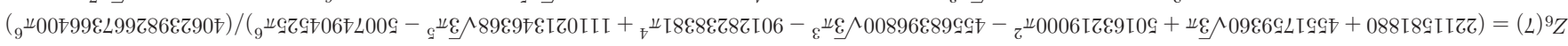

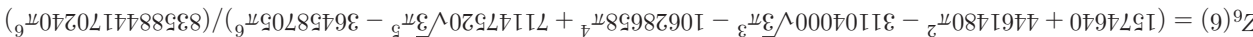

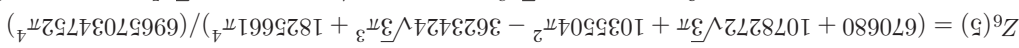

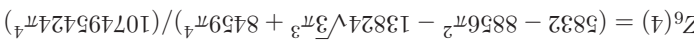

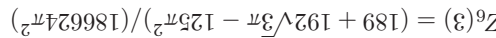

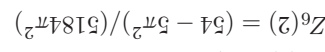


Open Access. This article is distributed under the terms of the Creative Commons Attribution License which permits any use, distribution and reproduction in any medium, provided the original author(s) and source are credited.

\section{References}

[1] O. Aharony, O. Bergman, D.L. Jafferis and J. Maldacena, $N=6$ superconformal Chern-Simons-matter theories, M2-branes and their gravity duals, JHEP 10 (2008) 091 [arXiv:0806.1218] [INSPIRE].

[2] A. Kapustin, B. Willett and I. Yaakov, Exact results for Wilson loops in superconformal Chern-Simons theories with matter, JHEP 03 (2010) 089 [arXiv: 0909.4559] [INSPIRE].

[3] D.L. Jafferis, The exact superconformal R-symmetry extremizes Z, JHEP 05 (2012) 159 [arXiv: 1012.3210] [INSPIRE].

[4] N. Hama, K. Hosomichi and S. Lee, Notes on SUSY gauge theories on three-sphere, JHEP 03 (2011) 127 [arXiv: 1012.3512] [INSPIRE].

[5] V. Pestun, Localization of gauge theory on a four-sphere and supersymmetric Wilson loops, Commun. Math. Phys. 313 (2012) 71 [arXiv:0712.2824] [InSPIRE].

[6] N. Drukker, M. Mariño and P. Putrov, From weak to strong coupling in ABJM theory, Commun. Math. Phys. 306 (2011) 511 [arXiv:1007.3837] [InSPIRE].

[7] I.R. Klebanov and A.A. Tseytlin, Entropy of near extremal black p-branes, Nucl. Phys. B 475 (1996) 164 [hep-th/9604089] [INSPIRE].

[8] H. Fuji, S. Hirano and S. Moriyama, Summing up all genus free energy of ABJM matrix model, JHEP 08 (2011) 001 [arXiv:1106.4631] [INSPIRE].

[9] M. Mariño and P. Putrov, ABJM theory as a Fermi gas, J. Stat. Mech. 1203 (2012) P03001 [arXiv: 1110.4066] [INSPIRE].

[10] M. Mariño and P. Putrov, Interacting fermions and $N=2$ Chern-Simons-matter theories, arXiv: 1206.6346 [INSPIRE].

[11] A. Klemm, M. Mariño, M. Schiereck and M. Soroush, ABJM Wilson loops in the Fermi gas approach, arXiv:1207.0611 [INSPIRE].

[12] H. Ooguri, C. Vafa and E.P. Verlinde, Hartle-Hawking wave-function for flux compactifications, Lett. Math. Phys. 74 (2005) 311 [hep-th/0502211] [INSPIRE].

[13] Y. Hatsuda, S. Moriyama and K. Okuyama, Exact results on the ABJM Fermi gas, JHEP 10 (2012) 020 [arXiv: 1207.4283] [INSPIRE].

[14] P. Putrov and M. Yamazaki, Exact ABJM partition function from TBA, Mod. Phys. Lett. A 27 (2012) 1250200 [arXiv:1207.5066] [InSPIRE].

[15] A. Cagnazzo, D. Sorokin and L. Wulff, String instanton in $A d S_{4} \times C P^{3}$, JHEP 05 (2010) 009 [arXiv:0911.5228] [INSPIRE].

[16] N. Drukker, M. Mariño and P. Putrov, Nonperturbative aspects of ABJM theory, JHEP 11 (2011) 141 [arXiv:1103.4844] [INSPIRE].

[17] C.A. Tracy and H. Widom, Proofs of two conjectures related to the thermodynamic Bethe ansatz, Commun. Math. Phys. 179 (1996) 667 [solv-int/9509003] [INSPIRE]. 
[18] K. Okuyama, A note on the partition function of ABJM theory on $S^{3}$, Prog. Theor. Phys. 127 (2012) 229 [arXiv:1110.3555] [INSPIRE].

[19] M. Hanada, M. Honda, Y. Honma, J. Nishimura, S. Shiba, et al., Numerical studies of the ABJM theory for arbitrary $N$ at arbitrary coupling constant, JHEP 05 (2012) 121 [arXiv:1202.5300] [INSPIRE].

[20] M. Mariño and P. Putrov, Exact results in ABJM theory from topological strings, JHEP 06 (2010) 011 [arXiv:0912.3074] [INSPIRE].

[21] O. Aharony, O. Bergman and D.L. Jafferis, Fractional M2-branes, JHEP 11 (2008) 043 [arXiv: 0807.4924] [INSPIRE].

[22] M. Mariño, Chern-Simons theory, matrix integrals and perturbative three manifold invariants, Commun. Math. Phys. 253 (2004) 25 [hep-th/0207096] [INSPIRE].

[23] M. Aganagic, A. Klemm, M. Mariño and C. Vafa, Matrix model as a mirror of Chern-Simons theory, JHEP 02 (2004) 010 [hep-th/0211098] [INSPIRE].

[24] N. Drukker and D. Trancanelli, A supermatrix model for $N=6$ super Chern-Simons-matter theory, JHEP 02 (2010) 058 [arXiv: 0912.3006] [INSPIRE].

[25] R. Gopakumar and C. Vafa, M theory and topological strings. 2., hep-th/9812127 [INSPIRE].

[26] E. Witten, Quantum background independence in string theory, hep-th/9306122 [INSPIRE].

[27] S.H. Katz, A. Klemm and C. Vafa, M theory, topological strings and spinning black holes, Adv. Theor. Math. Phys. 3 (1999) 1445 [hep-th/9910181] [InSPIRE].

[28] M. Aganagic, M. Mariño and C. Vafa, All loop topological string amplitudes from Chern-Simons theory, Commun. Math. Phys. 247 (2004) 467 [hep-th/0206164] [InSPIRE].

[29] S. Bhattacharyya, A. Grassi, M. Mariño and A. Sen, A one-loop test of quantum supergravity, arXiv:1210.6057 [INSPIRE].

[30] G. Lockhart and C. Vafa, Superconformal partition functions and non-perturbative topological strings, arXiv:1210.5909 [INSPIRE]. 